\title{
CONSISTENT LOCAL PROJECTION STABILIZED FINITE ELEMENT METHODS
}

\author{
GABRIEL R. BARRENECHEA AND FRÉDÉRIC VALENTIN
}

\begin{abstract}
This work establishes a formal derivation of local projection stabilized methods as a result of an enriched Petrov-Galerkin strategy for the Stokes problem. Both velocity and pressure finite element spaces are enhanced with solutions of residual-based local problems, and then the static condensation procedure is applied to derive new methods. The approach keeps degrees of freedom unchanged while gives rise to new stable and consistent methods for continuous and discontinuous approximation spaces for the pressure. The resulting methods do not need the use of a macro-element grid structure and are parameter-free. The numerical analysis is carried out showing optimal convergence in natural norms, and moreover, two ways of rendering the velocity field locally mass conservative are proposed. Some numerics validate the theoretical results.
\end{abstract}

\section{INTRODUCTION}

Residual of Euler-Lagrange equations have been involved in the the construction of finite element methods as a way to include the most desirable pair of spaces, namely the simplest and equal order elements, in the set of stable methods for the Stokes equation. Often adopted as an error indicator, residuals have the important property to vanish when finite solutions approach the exact ones. Thereby, when added to the standard Galerkin method, residualdependent terms keep the approach consistent and the resulting methods, called stabilized methods, achieve optimal convergence rates.

Now, stabilized finite element methods for the Stokes problem have been criticized because of their need to set a constant, called the stabilization parameter. Some alternatives have been proposed to set up this constant among which is the enriching space approach. The idea is to enhance a pair of non stable finite element spaces and look for a discrete solution in the underlying augmented stable spaces through the standard Galerkin method. What drives the choice of additional basis functions is to fulfill the inf-sup condition without increasing the size of the corresponding linear system, i.e, without incorporating extra degrees of freedom. A sufficient condition to respect the latter constraint is to perform static condensation

Key words and phrases. pressure polynomial projection methods, Stokes problem, local problem, consistent method. 
procedure at the local level. This leads the enriched part of the numerical solution to be defined in terms of residuals and naturally incorporate them into the method. Moreover, this immediately establishes a bridge with stabilized methods where stabilized parameters are defined once and for all with respect to the integral of enriching basis functions. For instance, the equal order linear pair of spaces is made stable by adding bubble functions (resulting in the so-called mini-element, [3]) which might be related to a stabilized finite element method (see [21]). Ever since, literature on the subject has been steadily growing with the introduction of new techniques as the Residual-Free-Bubbles (RFB) [10, 9], the Variational Multiscale Methods (VMS) [19], and recently Petrov-Galerkin Enriched Methods (PGEM) $[15,4]$, just to name a few.

As an alternative to avoid this constant to set, in [13] a new type of methods, called polynomial pressure projection stabilized methods, have been developed (see also [7], where the analysis has been carried out). In fact, a parameter-free new stabilization term, based on the penalization of some projection error on the pressure, is added to the formulation in order to stabilize it. More precisely, if $p_{l}$ is the discrete polynomial pressure of order $l$, the method adds to the Galerkin method the following term (see next section for notations):

$$
\sum_{K \in \mathcal{T}_{h}} \frac{1}{\nu}\left(p_{l}-\rho_{l-1}\left(p_{l}\right), q_{l}-\rho_{l-1}\left(q_{l}\right)\right)_{K},
$$

where $\rho_{l-1}\left(q_{l}\right)$ is the local $L^{2}$ projection of $q_{l}$ onto space $\mathbb{P}_{l-1}(K)$ and $\nu \in \mathbb{R}^{+}$. The stated method can be seen as belonging to the class of Local Projection Stabilized (LPS) methods first introduced in [6]. Although similar, the method in [6] differs from the ones proposed in [13] since, rather than been based on fluctuations of the pressure, it involves the fluctuations of the gradient of pressure, and demands a parameter to be fixed. Some alternative forms of LPS methods have been proposed and analyzed inside a two-level approach (i.e., a dual coarser mesh is needed) [11], or based on an one-level strategy in [17] (see also [22] for a recent review and further references). What is common among all LPS methods is their lack of consistency, and the general strategy has been to prove that this remains bounded within the discretization error.

Now, none of the above mentioned works provides a systematic way of recovering these methods as the result of an enrichment strategy of the finite element space, although some hints on this may be found in [8]. This work aims then at proposing a way to formally derive this type of methods in the PGEM framework. In particular, we focus on the methods proposed in [13] as the LPS gradient-based versions still depend on fixing parameters. We enrich both the velocity and pressure spaces and characterize the enriched part as the solution 
of local problems. Then, we apply the static condensation procedure to obtain new stabilized finite element methods which are now consistent (or weakly consistent for low order velocity approximations) and parameter free. In this work the extra term reads

$$
\sum_{K \in \mathcal{T}_{h}} \frac{1}{\nu}\left(p_{l}-\rho_{0}\left(p_{l}\right)+p_{e}^{M}\left(\boldsymbol{f}-\nu \Delta \boldsymbol{u}_{k}\right), q_{l}-\rho_{0}\left(q_{l}\right)\right)_{K},
$$

plus appropriate jump terms if we use discontinuous pressure approximations, where $\boldsymbol{u}_{k}$ stands for an order $k$ polynomial velocity and $p_{e}^{M}\left(\boldsymbol{f}-\nu \Delta \boldsymbol{u}_{k}\right)$ belongs to $L_{0}^{2}(K)$ and solves a local Stokes problem (see Section 2 for further details). At this point we just remark that the shape of the added terms prevents us from using the analysis from [11] since in our case the stabilization terms are no longer equivalent to $\sum_{K \in \mathcal{T}_{h}} h_{K}^{2}\left\|\nabla p_{l}\right\|_{0, K}^{2}$.

When applied to equal-order linear continuous interpolations for velocity and pressure we recover one of the methods given in [13] with a modified right hand side (in the case the pressure is approached using piecewise constant functions the method coincides with one of the methods proposed in [1]). Furthermore, this process gives a completely new method if the pressure is approximated using discontinuous linear functions. This method does not need a two-level strategy and also all the computations may be done at the element level, at the cost of enlarging the stencil of the matrix since now new jump terms appear in order to stabilize the discontinuities of the pressure. It is worth saying that jump terms (of a different nature) were already present in the LPS framework in [11] when discontinuous approximations of the pressure were used. We prove the method induces a positive definite linear system and leads to optimal convergence in natural norms.

Finally, the enrichment strategy suggests us two different ways to recover a locally mass conservative velocity field, an issue usually overlooked when solving the Stokes problem in the stabilized finite element context, but vital when it comes to couple the Stokes (or NavierStokes) equation with a heat or transport equation. We prove this fact and also prove that the addition of this new velocity field does not undermine the convergence of the method.

The plan of the paper is as follows: we end this section with some notations and definitions to be used throughout this manuscript. Next section is devoted to the presentation of the enriching space strategy and ends with the final form of the stabilized method. Section 3 exhibits the methods for three different choices of pairs of finite elements and includes a wellposedness result and error estimates for the new one. Numerical validations are in Section 4 and some conclusions are drawn in Section 5.

1.1. Preliminary notations. Let $(\cdot, \cdot)_{D}$ be the inner product in $L^{2}(D)$ (or in $L^{2}(D)^{2}$ or $L^{2}(D)^{2 \times 2}$, when necessary), and we denote by $\|\cdot\|_{s, D}\left(|\cdot|_{s, D}\right)$ the norm (seminorm) in $H^{s}(D)$ 
(or $H^{s}(D)^{2}$, if necessary). As usual, $H^{0}(D)=L^{2}(D)$, and $|\cdot|_{0, D}=\|\cdot\|_{0, D}$. Also, $H($ div,$D)$ stands for the space of $L^{2}(D)^{2}$ functions whose divergence belongs to $L^{2}(D)$, provided with the norm

$$
\|\boldsymbol{v}\|_{d i v, D}:=\left\{\|\boldsymbol{v}\|_{0, D}^{2}+\|\nabla \cdot \boldsymbol{v}\|_{0, D}^{2}\right\}^{\frac{1}{2}} .
$$

Let $\Omega$ be an open bounded domain in $\mathbb{R}^{2}$ with polygonal boundary. A family of regular triangulations of $\Omega$ reads $\left\{\mathcal{T}_{h}\right\}_{h>0}$, built up using triangles $K$ with boundary $\partial K$, characteristic length $h_{K}:=\operatorname{diam}(K)$ and we denote $h:=\max \left\{h_{K}: K \in \mathcal{T}_{h}\right\}$. The set of internal edges $F$ of the triangulation is denoted by $\mathcal{E}_{h}$ with $h_{F}=|F|$.

We denote $\boldsymbol{n}$ the normal outward vector on $\partial K, \partial_{\boldsymbol{s}}$ and $\partial_{\boldsymbol{n}}$ the tangential and normal derivative operators, respectively, $\llbracket v \rrbracket$ stands for the jump of $v$ across $F, \Pi_{S}$ (denoted by $\rho_{0}$ in the introduction), where $S \subset \mathbb{R}^{2}$, is the orthogonal projection onto the constant space, i.e.,

$$
\Pi_{S}(q):=\frac{(q, 1)_{S}}{|S|}
$$

and $\mathbf{I}$ is the $\mathbb{R}^{2 \times 2}$ identity matrix.

In what follows $V_{h}$ stands for the usual finite element space of continuous piecewise linear polynomials with zero trace on $\partial \Omega$, and $\mathbf{V}_{h}:=\left[V_{h}\right]^{2}$. Also, $Q_{h}^{l}$ denotes the space of piecewise polynomials of degree $l, 0 \leq l \leq 1$, which is continuous or discontinuous in $\Omega$, and belong to $L_{0}^{2}(\Omega)$. In addition, it will be useful in the sequel the orthogonal complement of $Q_{h}^{l}$ in $L_{0}^{2}\left(\mathcal{T}_{h}\right)$ denoted here by $G_{h}$, where we define the following broken spaces

$$
\begin{aligned}
H_{0}^{1}\left(\mathcal{T}_{h}\right) & :=\left\{v:\left.v\right|_{K} \in H_{0}^{1}(K) \forall K \in \mathcal{T}_{h}\right\}, \\
L_{0}^{2}\left(\mathcal{T}_{h}\right) & :=\left\{v:\left.v\right|_{K} \in L_{0}^{2}(K) \forall K \in \mathcal{T}_{h}\right\} .
\end{aligned}
$$

\section{THE MODEL PROBLEM AND THE GENERAL FRAMEWORK}

Let us consider the following Stokes problem:

$$
\begin{aligned}
-\nu \Delta \boldsymbol{u}+\nabla p=\boldsymbol{f}, & \nabla \cdot \boldsymbol{u}=0 \quad \text { in } \Omega, \\
\boldsymbol{u} & =\mathbf{0} \quad \text { on } \partial \Omega,
\end{aligned}
$$

where $\nu \in \mathbb{R}^{+}$is the fluid viscosity and $\boldsymbol{f} \in L^{2}(\Omega)^{2}$.

The continuous weak form of problem (2) over spaces $\left[H_{0}^{1}(\Omega)^{2} \times L_{0}^{2}(\Omega)\right]$ satisfies the inf-sup condition and admits an unique solution [18]. This feature is not shared by its discrete version relied on the Galerkin method onto most of desirable pair of polynomial subspaces. Indeed, the simplest element $\mathbb{P}_{1} / \mathbb{P}_{0}$ or still the linear equal order space $\mathbb{P}_{1} / \mathbb{P}_{1}$ (either continuos

or discontinuous pressure cases) are out of reach. In what follows we intent to make the 
underlined pairs of spaces inf-sup stable through projection stabilized methods built up inside an enriching space strategy.

To this end, we start assuming that the design of interpolation basis functions must involve elemental residual of strong equations so as to incorporate missed features of exact solutions. This is not a strange approach as the whole class of stabilized and variational multiscale (inside which we include the RFB) methods are strongly based on this. What makes the present approach new is the way to involve residuals in the construction of basis functions which ultimately leads to a family of consistent stabilized projection methods.

Let us begin with a given initial unstable pair of interpolation spaces $\mathbf{V}_{h} \times Q_{h}^{l}$ as the trial and test spaces. By initial we mean that actual trial and test spaces are to be proposed enhancing $\mathbf{V}_{h} \times Q_{h}^{l}$. Since at this point no indication is available on how to set up enriched spaces, we augment initial space as "big" as we can. As for the enriched trial spaces we perform it selecting

$$
\left[\mathbf{V}_{h}+H_{0}^{1}(\Omega)^{2}\right] \times\left[Q_{h}^{l}+L_{0}^{2}\left(\mathcal{T}_{h}\right)\right]
$$

thus the exact solution $(\boldsymbol{u}, p)$ is approached by

$$
\left(\boldsymbol{u}_{h}, p_{h}\right):=\left(\boldsymbol{u}_{1}+\boldsymbol{u}_{e}, p_{l}+p_{e}\right)
$$

where $\left(\boldsymbol{u}_{1}, p_{l}\right) \in \mathbf{V}_{h} \times Q_{h}^{l}$ and $\left(\boldsymbol{u}_{e}, p_{e}\right) \in H_{0}^{1}(\Omega)^{2} \times L_{0}^{2}\left(\mathcal{T}_{h}\right)$. In regard to the enriched test spaces, we set it up through the direct sum

$$
\left[\mathbf{V}_{h} \oplus H_{0}^{1}\left(\mathcal{T}_{h}\right)^{2}\right] \times\left[Q_{h}^{l} \oplus G_{h}\right]
$$

This choice makes a function $\left(\boldsymbol{v}_{h}, q_{h}\right)$ in it uniquely decomposed into $\left(\boldsymbol{v}_{1}+\boldsymbol{v}_{b}, q_{l}+q_{b}\right)$, with $\left(\boldsymbol{v}_{1}, q_{l}\right) \in \mathbf{V}_{h} \times Q_{h}^{l}$ and $\left(\boldsymbol{v}_{b}, q_{b}\right) \in H_{0}^{1}\left(\mathcal{T}_{h}\right)^{2} \times G_{h}$.

Remark. Trial and test approximation spaces for pressure coincide for $l=0$. Conformity of enriched velocity is assured imposing continuity of $\boldsymbol{u}_{e}$ through a non homogenous transmission condition on the internal edges (see equations (6)-(7)). In addition, the test space for the velocity is also conforming and test and trial spaces always differ to one another.

Next, we propose the following Petrov-Galerkin scheme for $(2)$ : Find $\left(\boldsymbol{u}_{h}, p_{h}\right) \in\left[\mathbf{V}_{h}+\right.$ $\left.H_{0}^{1}(\Omega)^{2}\right] \times\left[Q_{h}^{l}+L_{0}^{2}\left(\mathcal{T}_{h}\right)\right]$ such that

$$
\nu\left(\nabla \boldsymbol{u}_{h}, \nabla \boldsymbol{v}_{h}\right)_{\Omega}-\left(p_{h}, \nabla \cdot \boldsymbol{v}_{h}\right)_{\Omega}+\left(q_{h}, \nabla \cdot \boldsymbol{u}_{h}\right)_{\Omega}=\left(\boldsymbol{f}, \boldsymbol{v}_{h}\right)_{\Omega}
$$


for all $\boldsymbol{v}_{h} \in \mathbf{V}_{h} \oplus H_{0}^{1}\left(\mathcal{T}_{h}\right)^{2}$ and all $q_{h} \in Q_{h} \oplus G_{h}$. It follows straight from the Petrov-Galerkin scheme that it is equivalent to the following system:

$$
\begin{gathered}
\nu\left(\nabla \boldsymbol{u}_{h}, \nabla \boldsymbol{v}_{1}\right)_{\Omega}-\left(p_{h}, \nabla \cdot \boldsymbol{v}_{1}\right)_{\Omega}+\left(q_{l}, \nabla \cdot \boldsymbol{u}_{h}\right)_{\Omega}=\left(\boldsymbol{f}, \boldsymbol{v}_{1}\right)_{\Omega} \quad \forall\left(\boldsymbol{v}_{1}, q_{l}\right) \in \mathbf{V}_{h} \times Q_{h}^{l} \\
\nu\left(\nabla \boldsymbol{u}_{h}, \nabla \boldsymbol{v}_{b}\right)_{K}-\left(p_{h}, \nabla \cdot \boldsymbol{v}_{b}\right)_{K}+\left(q_{b}, \nabla \cdot \boldsymbol{u}_{h}\right)_{K}=\left(\boldsymbol{f}, \boldsymbol{v}_{b}\right)_{K}
\end{gathered}
$$

for all $\left(\boldsymbol{v}_{b}, q_{b}\right) \in H_{0}^{1}(K)^{2} \times G_{h}, \forall K \in \mathcal{T}_{h}$. Since $\left.\nabla \cdot \boldsymbol{u}_{1}\right|_{K} \in \mathbb{R}$ and $\left.\boldsymbol{v}_{b}\right|_{\partial K}=0$, and using a standard argument, equation (4) above corresponds to the weak form of the problem

$$
-\nu \Delta \boldsymbol{u}_{e}+\nabla p_{e}=\boldsymbol{f}+\nu \Delta \boldsymbol{u}_{1}-\nabla p_{l} \quad, \quad \nabla \cdot \boldsymbol{u}_{e} \in \mathbb{P}_{l}(K) \quad \text { in } K,
$$

where the residual of the first equation defines the right hand side. Now, to close this differential problem we start by fixing the boundary conditions on $\boldsymbol{u}_{e}$. To this end we impose the following boundary condition on $\boldsymbol{u}_{e}$ :

$$
\boldsymbol{u}_{e}=\boldsymbol{g}_{e} \quad \text { on each } F \subset \partial K
$$

where $\boldsymbol{g}_{e}=\mathbf{0}$ if $F \subset \partial \Omega$, and $\boldsymbol{g}_{e}$ is the solution of

$$
\begin{aligned}
-\nu \partial_{\boldsymbol{s s}} \boldsymbol{g}_{e} & =\frac{\alpha}{h_{F}} \Pi_{F}\left(\llbracket \nu \partial_{\boldsymbol{n}} \boldsymbol{u}_{1}+p_{l} \mathbf{I} \cdot \boldsymbol{n} \rrbracket\right) \text { in } F, \\
\boldsymbol{g}_{e} & =\mathbf{0} \text { at the nodes }
\end{aligned}
$$

on the internal edges, where $\alpha \geq 0$. This boundary condition keeps the approach conforming and incorporates edge residuals close to those from the a posteriori error estimate given in $[2]$.

Remark. The choice $\alpha=0$ implies that the enrichment space for the velocity is composed of bubble functions which will turn out to be the correct way to stabilize continuous pressure spaces. For the case of discontinuous pressure spaces this constant will be set to $\alpha=1$.

Let us turn back to the local Stokes problems to compute $\left(\boldsymbol{u}_{e}, p_{e}\right)$. It emerges from (5) and $(7)$ that $\left(\boldsymbol{u}_{e}, p_{e}\right)$ inherits the degrees of freedom of $\left(\boldsymbol{u}_{1}, p_{l}\right)$ and that it might be split into $\left(\boldsymbol{u}_{e}, p_{e}\right)=\left(\boldsymbol{u}_{e}^{M}, p_{e}^{M}\right)+\left(\boldsymbol{u}_{e}^{G}, p_{e}^{G}\right)+\left(\boldsymbol{u}_{e}^{D}, p_{e}^{D}\right)$ where each contribution satisfies, respectively,

$$
\begin{aligned}
-\nu \Delta \boldsymbol{u}_{e}^{M}+\nabla p_{e}^{M}=\boldsymbol{f}+\nu \Delta \boldsymbol{u}_{1}-\nabla p_{l} \quad, \quad \nabla \cdot \boldsymbol{u}_{e}^{M}=0 \quad \text { in } K \\
\boldsymbol{u}_{e}^{M}=\mathbf{0} \quad \text { on } \partial K, \\
-\nu \Delta \boldsymbol{u}_{e}^{G}+\nabla p_{e}^{G}=\mathbf{0} \quad, \quad \nabla \cdot \boldsymbol{u}_{e}^{G} \in \mathbb{P}_{l}(K) \quad \text { in } K \\
\boldsymbol{u}_{e}^{G}=\mathbf{0} \quad \text { on } \partial K,
\end{aligned}
$$


and

$$
\begin{gathered}
-\nu \Delta \boldsymbol{u}_{e}^{D}+\nabla p_{e}^{D}=\mathbf{0} \quad, \quad \nabla \cdot \boldsymbol{u}_{e}^{D}=\frac{\int_{\partial K} \boldsymbol{g}_{e} \cdot \boldsymbol{n}}{|K|} \quad \text { in } K, \\
\boldsymbol{u}_{e}^{D}=\boldsymbol{g}_{e} \quad \text { on } \partial K .
\end{gathered}
$$

It remains to set problem (9). To this end, we first remark that, if $\left.\boldsymbol{f}\right|_{K}=\left(f_{1}, f_{2}\right)^{t} \in \mathbb{R}^{2}$, then the solution of (8) satisfies $\boldsymbol{u}_{e}^{M}=\mathbf{0}$ and $p_{e}^{M}=p_{e}^{M}\left(\boldsymbol{f}+\nu \Delta \boldsymbol{u}_{1}-\nabla p_{l}\right)$ is given by

$$
p_{e}^{M}=\boldsymbol{f} \cdot \mathbf{x}-\Pi_{K}(\boldsymbol{f} \cdot \mathbf{x})+p_{l}-\Pi_{K}\left(p_{l}\right) \in \mathbb{P}_{1}(K) .
$$

Hence, we reinforce the dependence of enriching functions in terms of residual by closing (9) as follows:

$$
\begin{array}{cl}
-\nu \Delta \boldsymbol{u}_{e}^{G}+\nabla p_{e}^{G}=\mathbf{0} \quad, \quad \nabla \cdot \boldsymbol{u}_{e}^{G}=-\frac{1}{\nu} p_{e}^{M}\left(\boldsymbol{f}+\nu \Delta \boldsymbol{u}_{1}-\nabla p_{l}\right) \quad \text { in } K, \\
\boldsymbol{u}_{e}^{G}=\mathbf{0} \quad \text { on } \partial K .
\end{array}
$$

It is worth remarking that $\left(\boldsymbol{u}_{e}, p_{e}\right)$ is uniquely defined through problems above and it satisfies equation (4). It remains then to fulfill equation (3). This, together with $\boldsymbol{u}_{e}^{M}=\mathbf{0}$, lead to our method given by: Find $\left(\boldsymbol{u}_{1}, p_{l}\right) \in \mathbf{V}_{h} \times Q_{h}^{l}$ such that

$$
\begin{gathered}
\nu\left(\nabla \boldsymbol{u}_{1}, \nabla \boldsymbol{v}_{1}\right)_{\Omega}+\nu \sum_{K \in \mathcal{T}_{h}}\left[\left(\nabla \boldsymbol{u}_{e}^{G}, \nabla \boldsymbol{v}_{1}\right)_{K}+\left(\nabla \boldsymbol{u}_{e}^{D}, \nabla \boldsymbol{v}_{1}\right)_{K}\right] \\
-\left(p_{l}, \nabla \cdot \boldsymbol{v}_{1}\right)_{\Omega}-\sum_{K \in \mathcal{T}_{h}}\left(p_{e}, \nabla \cdot \boldsymbol{v}_{1}\right)_{K}+\left(q_{l}, \nabla \cdot \boldsymbol{u}_{1}\right)_{\Omega} \\
+\sum_{K \in \mathcal{T}_{h}}\left[\left(q_{l}, \nabla \cdot \boldsymbol{u}_{e}^{G}\right)_{K}+\left(q_{l}, \nabla \cdot \boldsymbol{u}_{e}^{D}\right)_{K}\right]=\left(\boldsymbol{f}, \boldsymbol{v}_{1}\right)_{\Omega},
\end{gathered}
$$

for all $\left(\boldsymbol{v}_{1}, q_{l}\right) \in \mathbf{V}_{h} \times Q_{h}^{l}$.

Some of the terms in (13) can be simplified. First, integrating by parts and using that $\boldsymbol{u}_{e}^{G} \in H_{0}^{1}(K)^{2}$ and $\Delta \boldsymbol{v}_{1}=0$ in each $K$, we get

$$
\begin{aligned}
& \left(\nabla \boldsymbol{u}_{e}^{G}, \nabla \boldsymbol{v}_{1}\right)_{K}=0 \\
& \left(\nabla \boldsymbol{u}_{e}^{D}, \nabla \boldsymbol{v}_{1}\right)_{K}=\left(\boldsymbol{u}_{e}^{D}, \nu \partial_{\boldsymbol{n}} \boldsymbol{u}_{1}\right)_{\partial K}
\end{aligned}
$$

Furthermore, since $p_{e} \in L_{0}^{2}(K)$ we obtain

$$
\left(p_{e}, \nabla \cdot \boldsymbol{v}_{1}\right)_{K}=0
$$


and from the characterization (11) we easily see that

$$
\begin{aligned}
\left(q_{l}, \nabla \cdot \boldsymbol{u}_{e}^{G}\right)_{K} & =\frac{1}{\nu}\left(q_{l}, p_{l}-\Pi_{K}\left(p_{l}\right)\right)_{K}-\frac{1}{\nu}\left(q_{l}, p_{e}^{M}\left(\boldsymbol{f}+\nu \Delta \boldsymbol{u}_{1}\right)\right)_{K} \\
& =\frac{1}{\nu}\left(q_{l}-\Pi_{K}\left(q_{l}\right), p_{l}-\Pi_{K}\left(p_{l}\right)\right)_{K}-\frac{1}{\nu}\left(q_{l}-\Pi_{K}\left(q_{l}\right), p_{e}^{M}\left(\boldsymbol{f}+\nu \Delta \boldsymbol{u}_{1}\right)\right)_{K} .
\end{aligned}
$$

Finally, integrating by parts we further get

$$
\left(q_{l}, \nabla \cdot \boldsymbol{u}_{e}^{D}\right)_{K}=-\left(\nabla q_{l}, \boldsymbol{u}_{e}^{D}\right)_{K}+\left(\boldsymbol{u}_{e}^{D}, q_{l} \mathbf{I} \cdot \boldsymbol{n}\right)_{\partial K} .
$$

Gathering last results, we rewrite (13) as: Find $\left(\boldsymbol{u}_{1}, p_{l}\right) \in \mathbf{V}_{h} \times Q_{h}^{l}$ such that

$$
\begin{gathered}
\nu\left(\nabla \boldsymbol{u}_{1}, \nabla \boldsymbol{v}_{1}\right)_{\Omega}-\left(p_{l}, \nabla \cdot \boldsymbol{v}_{1}\right)_{\Omega}+\left(q_{l}, \nabla \cdot \boldsymbol{u}_{1}\right)_{\Omega} \\
+\frac{1}{\nu} \sum_{K \in \mathcal{T}_{h}}\left(p_{l}-\Pi_{K}\left(p_{l}\right)+p_{e}^{M}\left(-\nu \Delta \boldsymbol{u}_{1}\right), q_{l}-\Pi_{K}\left(q_{l}\right)\right)_{K}-\left(\nabla q_{l}, \boldsymbol{u}_{e}^{D}\right)_{K} \\
+\sum_{F \in \mathcal{E}_{h}}\left(\boldsymbol{u}_{e}^{D}, \llbracket \nu \partial_{\boldsymbol{n}} \boldsymbol{v}_{1}+q_{l} \mathbf{I} \cdot \boldsymbol{n} \rrbracket\right)_{F}=\left(\boldsymbol{f}, \boldsymbol{v}_{1}\right)_{\Omega}+\frac{1}{\nu} \sum_{K \in \mathcal{T}_{h}}\left(p_{e}^{M}(\boldsymbol{f}), q_{l}-\Pi_{K}\left(q_{l}\right)\right)_{K},
\end{gathered}
$$

for all $\left(\boldsymbol{v}_{1}, q_{l}\right) \in \mathbf{V}_{h} \times Q_{h}^{l}$.

The edge contribution in method (14) rewrites in a more convenient form following [1]. In fact, since $\left.\Pi_{F}\left(\llbracket \nu \partial_{\boldsymbol{n}} \boldsymbol{u}_{1}+p_{l} \mathbf{I} \cdot \boldsymbol{n} \rrbracket\right)\right|_{F}$ is a constant function, then $\boldsymbol{g}_{e}=\left.\alpha g_{F} \mathbf{I} \cdot \Pi_{F}\left(\llbracket \nu \partial_{\boldsymbol{n}} \boldsymbol{u}_{1}+p_{l} \mathbf{I} \cdot \boldsymbol{n} \rrbracket\right)\right|_{F}$, where $g_{F}$ stands for the solution of

$$
-\nu \partial_{s s} g_{F}=\frac{1}{h_{F}} \text { in } F, \quad g_{F}=0 \text { at the nodes }
$$

on the internal edges.

Remark. It turns out that the solution of (15) may be calculated explicitly, in fact, is a polynomial of degree two in each one of the edges $F$. Then, since $g_{F}$ vanishes on the endpoints of $F$, and, for $q \in \mathbb{P}_{1}(F)$ the function $q-\Pi_{F}(q)$ vanishes on the mid-point of $F$, using Simpson's rule we obtain

$$
\left(g_{F}, q\right)_{F}=\left(g_{F}, q-\Pi_{F}(q)\right)_{F}+\left(g_{F}, \Pi_{F}(q)\right)_{F}=\left(g_{F}, \Pi_{F}(q)\right)_{F}
$$

Using this last remark we realize that the edge terms read

$$
\begin{gathered}
\left(\boldsymbol{u}_{e}^{D}, \llbracket \nu \partial_{\boldsymbol{n}} \boldsymbol{v}_{1}+q_{l} \mathbf{I} \cdot \boldsymbol{n} \rrbracket\right)_{F}=\left.\left.\left[\int_{F} g_{F}\right] \alpha \Pi_{F}\left(\llbracket \nu \partial_{\boldsymbol{n}} \boldsymbol{u}_{1}+p_{l} \mathbf{I} \cdot \boldsymbol{n} \rrbracket\right)\right|_{F} \cdot \Pi_{F}\left(\llbracket \nu \partial_{\boldsymbol{n}} \boldsymbol{v}_{1}+q_{l} \mathbf{I} \cdot \boldsymbol{n} \rrbracket\right)\right|_{F} \\
=\frac{\left(g_{F}, 1\right)_{F}}{|F|} \alpha\left(\Pi_{F}\left(\llbracket \nu \partial_{\boldsymbol{n}} \boldsymbol{u}_{1}+p_{l} \mathbf{I} \cdot \boldsymbol{n} \rrbracket\right), \Pi_{F}\left(\llbracket \nu \partial_{\boldsymbol{n}} \boldsymbol{v}_{1}+q_{l} \mathbf{I} \cdot \boldsymbol{n} \rrbracket\right)\right)_{F}
\end{gathered}
$$

with

$$
\frac{\left(g_{F}, 1\right)_{F}}{|F|}=\frac{h_{F}}{12 \nu} .
$$


Therefore, using this rewriting of the edge terms (14) becomes: Find $\left(\boldsymbol{u}_{1}, p_{l}\right) \in \mathbf{V}_{h} \times Q_{h}^{l}$ such that

$$
\begin{gathered}
\nu\left(\nabla \boldsymbol{u}_{1}, \nabla \boldsymbol{v}_{1}\right)_{\Omega}-\left(p_{l}, \nabla \cdot \boldsymbol{v}_{1}\right)_{\Omega}+\left(q_{l}, \nabla \cdot \boldsymbol{u}_{1}\right)_{\Omega} \\
+\sum_{K \in \mathcal{T}_{h}}\left(\boldsymbol{u}_{e}^{D}, \nabla q_{l}\right)_{K}+\frac{1}{\nu} \sum_{K \in \mathcal{T}_{h}}\left(p_{l}-\Pi_{K}\left(p_{l}\right)+p_{e}^{M}\left(-\nu \Delta \boldsymbol{u}_{1}\right), q_{l}-\Pi_{K}\left(q_{l}\right)\right)_{K} \\
+\sum_{F \in \mathcal{E}_{h}} \frac{\alpha h_{F}}{12 \nu}\left(\Pi_{F}\left(\llbracket \nu \partial_{\boldsymbol{n}} \boldsymbol{u}_{1}+p_{l} \mathbf{I} \cdot \boldsymbol{n} \rrbracket\right), \Pi_{F}\left(\llbracket \nu \partial_{\boldsymbol{n}} \boldsymbol{v}_{1}+q_{l} \mathbf{I} \cdot \boldsymbol{n} \rrbracket\right)\right)_{F} \\
=\left(\boldsymbol{f}, \boldsymbol{v}_{1}\right)_{\Omega}+\frac{1}{\nu} \sum_{K \in \mathcal{T}_{h}}\left(p_{e}^{M}(\boldsymbol{f}), q_{l}-\Pi_{K}\left(q_{l}\right)\right)_{K}
\end{gathered}
$$

for all $\left(\boldsymbol{v}_{1}, q_{l}\right) \in \mathbf{V}_{h} \times Q_{h}^{l}$.

Next, we highlight the new method above for different choices of interpolation spaces and characterize them with respect to known stabilized and local projection methods.

\section{Applications}

3.1. The simplest element $\mathbb{P}_{1} \times \mathbb{P}_{0}$. In this case we consider $\alpha=1$ and then the method becomes: Find $\left(\boldsymbol{u}_{1}, p_{0}\right) \in \mathbf{V}_{h} \times Q_{h}^{0}$ such that

$$
\begin{gathered}
\nu\left(\nabla \boldsymbol{u}_{1}, \nabla \boldsymbol{v}_{1}\right)_{\Omega}-\left(p_{0}, \nabla \cdot \boldsymbol{v}_{1}\right)_{\Omega}+\left(q_{0}, \nabla \cdot \boldsymbol{u}_{1}\right)_{\Omega} \\
+\sum_{F \in \mathcal{E}_{h}} \frac{h_{F}}{12 \nu}\left(\llbracket \nu \partial_{\boldsymbol{n}} \boldsymbol{u}_{1}+p_{0} \mathbf{I} \cdot \boldsymbol{n} \rrbracket, \llbracket \nu \partial_{\boldsymbol{n}} \boldsymbol{v}_{1}+q_{0} \mathbf{I} \cdot \boldsymbol{n} \rrbracket\right)_{F}=\left(\boldsymbol{f}, \boldsymbol{v}_{1}\right)_{\Omega},
\end{gathered}
$$

for all $\left(\boldsymbol{v}_{1}, q_{0}\right) \in \mathbf{V}_{h} \times Q_{h}^{0}$, which is precisely one of the methods presented and analyzed in $[1]$.

3.2. The element $\mathbb{P}_{1} \times \mathbb{P}_{1}$ with continuous pressures. In this case we choose $\alpha=0$ (i.e., we enrich the trial space with bubble functions), and use the fact that $\boldsymbol{u}_{e}^{D}=\mathbf{0}$ to obtain the method: Find $\left(\boldsymbol{u}_{1}, p_{1}\right) \in \mathbf{V}_{h} \times Q_{h}^{1}$ such that

$$
\nu\left(\nabla \boldsymbol{u}_{1}, \nabla \boldsymbol{v}_{1}\right)_{\Omega}-\left(p_{1}, \nabla \cdot \boldsymbol{v}_{1}\right)_{\Omega}+\left(q_{1}, \nabla \cdot \boldsymbol{u}_{1}\right)_{\Omega}
$$

$+\frac{1}{\nu} \sum_{K \in \mathcal{T}_{h}}\left(p_{1}-\Pi_{K}\left(p_{1}\right)+p_{e}^{M}\left(-\nu \Delta \boldsymbol{u}_{1}\right), q_{1}-\Pi_{K}\left(q_{1}\right)\right)_{K}=\left(\boldsymbol{f}, \boldsymbol{v}_{1}\right)_{\Omega}+\frac{1}{\nu} \sum_{K \in \mathcal{T}_{h}}\left(p_{e}^{M}(\boldsymbol{f}), q_{1}-\Pi_{K}\left(q_{1}\right)\right)_{K}$,

for all $\left(\boldsymbol{v}_{1}, q_{1}\right) \in \mathbf{V}_{h} \times Q_{h}^{1}$. This method has a similar structure to one of the methods analyzed in [7] although, unlike [7], the method (18) has been derived in a residual-based framework. The analysis of (18) is covered by the more general case presented in the next section, and so, we omit it here. 
3.3. The element $\mathbb{P}_{1} \times \mathbb{P}_{1}^{\text {disc }}$. The space of discrete pressures contains now discontinuous functions. Then, considering $\alpha>0$ the method reads: Find $\left(\boldsymbol{u}_{1}, p_{1}\right) \in \mathbf{V}_{h} \times Q_{h}^{1}$ such that

$$
\begin{gathered}
\nu\left(\nabla \boldsymbol{u}_{1}, \nabla \boldsymbol{v}_{1}\right)_{\Omega}-\left(p_{1}, \nabla \cdot \boldsymbol{v}_{1}\right)_{\Omega}+\left(q_{1}, \nabla \cdot \boldsymbol{u}_{1}\right)_{\Omega} \\
+\frac{1}{\nu} \sum_{K \in \mathcal{T}_{h}}\left(p_{1}-\Pi_{K}\left(p_{1}\right)+p_{e}^{M}\left(-\nu \Delta \boldsymbol{u}_{1}\right), q_{1}-\Pi_{K}\left(q_{1}\right)\right)_{K}+\sum_{K \in \mathcal{T}_{h}}\left(\boldsymbol{u}_{e}^{D}, \nabla q_{1}\right)_{K} \\
+\sum_{F \in \mathcal{E}_{h}} \frac{\alpha h_{F}}{12 \nu}\left(\Pi_{F}\left(\llbracket \nu \partial_{\boldsymbol{n}} \boldsymbol{u}_{1}+p_{1} \mathbf{I} \cdot \boldsymbol{n} \rrbracket\right), \Pi_{F}\left(\llbracket \nu \partial_{\boldsymbol{n}} \boldsymbol{v}_{1}+q_{1} \mathbf{I} \cdot \boldsymbol{n} \rrbracket\right)\right)_{F} \\
=\left(\boldsymbol{f}, \boldsymbol{v}_{1}\right)_{\Omega}+\frac{1}{\nu} \sum_{K \in \mathcal{T}_{h}}\left(p_{e}^{M}(\boldsymbol{f}), q_{1}-\Pi_{K}\left(q_{1}\right)\right)_{K},
\end{gathered}
$$

for all $\left(\boldsymbol{v}_{1}, q_{1}\right) \in \mathbf{V}_{h} \times Q_{h}^{1}$. For reasons that will be detailed in the appendix, for $\alpha$ small enough we can neglect the term $\sum_{K \in \mathcal{T}_{h}}\left(\boldsymbol{u}_{e}^{D}, \nabla q_{1}\right)_{K}$ and propose the following final form of the method: Find $\left(\boldsymbol{u}_{1}, p_{1}\right) \in \mathbf{V}_{h} \times Q_{h}^{1}$ such that

$$
\mathbf{B}\left(\left(\boldsymbol{u}_{1}, p_{1}\right),\left(\boldsymbol{v}_{1}, q_{1}\right)\right)=\left(\boldsymbol{f}, \boldsymbol{v}_{1}\right)_{\Omega}+\frac{1}{\nu} \sum_{K \in \mathcal{T}_{h}}\left(p_{e}^{M}(\boldsymbol{f}), q_{1}-\Pi_{K}\left(q_{1}\right)\right)_{K},
$$

for all $\left(\boldsymbol{v}_{1}, q_{1}\right) \in \mathbf{V}_{h} \times Q_{h}^{1}$, where

$$
\begin{aligned}
\mathbf{B}\left(\left(\boldsymbol{u}_{1}, p_{1}\right),\left(\boldsymbol{v}_{1}, q_{1}\right)\right) & :=\nu\left(\nabla \boldsymbol{u}_{1}, \nabla \boldsymbol{v}_{1}\right)_{\Omega}-\left(p_{1}, \nabla \cdot \boldsymbol{v}_{1}\right)_{\Omega}+\left(q_{1}, \nabla \cdot \boldsymbol{u}_{1}\right)_{\Omega} \\
& +\frac{1}{\nu} \sum_{K \in \mathcal{T}_{h}}\left(p_{1}-\Pi_{K}\left(p_{1}\right)+p_{e}^{M}\left(-\nu \Delta \boldsymbol{u}_{1}\right), q_{1}-\Pi_{K}\left(q_{1}\right)\right)_{K} \\
& +\sum_{F \in \mathcal{E}_{h}} \frac{\alpha h_{F}}{12 \nu}\left(\Pi_{F}\left(\llbracket \nu \partial_{\boldsymbol{n}} \boldsymbol{u}_{1}+p_{1} \mathbf{I} \cdot \boldsymbol{n} \rrbracket\right), \Pi_{F}\left(\llbracket \nu \partial_{\boldsymbol{n}} \boldsymbol{v}_{1}+q_{1} \mathbf{I} \cdot \boldsymbol{n} \rrbracket\right)\right)_{F} .
\end{aligned}
$$

Remark. The restriction on the value of $\alpha$ is done only to rigorously prove the link between the method and the enrichment strategy, so, from now on, we will consider $\alpha=1$ (which is the value used also in the numerical experiments). As a matter of fact, in the numerical experiments we have remarked virtually no impact of the value of $\alpha$ in the overall errors, and hence the choice $\alpha=1$ is completely justified.

Remark. The stated methods in this work may be classified as members of LPS family as long as we adopt low order pairs of spaces. As a matter of fact, when it comes to use them along with higher order polynomial interpolation such nomenclature is less clear as extra weak terms include the function $p_{e}^{M}\left(-\nu \Delta \boldsymbol{u}_{k}\right)$ (responsible for making methods strongly consistent) instead of subtracting a $L^{2}$ projection of pressure onto lower order finite element subspaces [13]. This new avenue deserves further investigation and will be exploited in forthcoming works. 
3.4. Error analysis. The well-posedenees and consistency of the method are proved first. Let $\|\cdot\|_{h}$ be the mesh-dependent norm given by

$$
\left\|\left(\boldsymbol{v}_{1}, q_{1}\right)\right\|_{h}^{2}:=\nu\left|\boldsymbol{v}_{1}\right|_{1, \Omega}^{2}+\frac{1}{\nu} \sum_{K \in \mathcal{T}_{h}}\left\|q_{1}-\Pi_{K}\left(q_{1}\right)\right\|_{0, K}^{2}+\sum_{F \in \mathcal{E}_{h}} \frac{h_{F}}{12 \nu}\left\|\Pi_{F}\left(\llbracket \nu \partial_{\boldsymbol{n}} \boldsymbol{v}_{1}+q_{1} \mathbf{I} \cdot \boldsymbol{n} \rrbracket\right)\right\|_{0, F}^{2} .
$$

Then we present the following result.

Lemma 1. For all $\left(\boldsymbol{v}_{1}, q_{1}\right) \in \mathbf{V}_{h} \times Q_{h}^{1}$ there holds

$$
\mathbf{B}\left(\left(\boldsymbol{v}_{1}, q_{1}\right),\left(\boldsymbol{v}_{1}, q_{1}\right)\right)=\left\|\left(\boldsymbol{v}_{1}, q_{1}\right)\right\|_{h}^{2},
$$

and (21) has a unique solution $\left(\boldsymbol{u}_{1}, p_{1}\right) \in \mathbf{V}_{h} \times Q_{h}^{1}$. Let also $(\boldsymbol{u}, p) \in H^{2}(\Omega)^{2} \times H^{1}(\Omega)$ be the solution of $(2)$ and $\left(\boldsymbol{u}_{1}, p_{1}\right)$ the solution of $(21)$. Then

$$
\mathbf{B}\left(\left(\boldsymbol{u}-\boldsymbol{u}_{1}, p-p_{1}\right),\left(\boldsymbol{v}_{1}, q_{1}\right)\right)=0
$$

for all $\left(\boldsymbol{v}_{1}, q_{1}\right) \in \mathbf{V}_{h} \times Q_{h}^{1}$.

Proof. The first part is immediate from the definition of the bilinear form $\mathbf{B}(.,$.$) in (22)$. For the consistency result we note that since $\boldsymbol{u} \in H^{2}(\Omega)^{2}$ and $p \in H^{1}(\Omega)$ then $\llbracket \partial_{\boldsymbol{n}} \boldsymbol{u} \rrbracket=\mathbf{0}$ and $\llbracket p \rrbracket=0$, and the jump terms vanish. The consistency result follows recalling that $p-\Pi_{K}(p)=p_{e}^{M}(\nabla p)$, and so the element wise enriching function $p-\Pi_{K}(p)+p_{e}^{M}(\boldsymbol{f}-\nu \Delta \boldsymbol{u})$ in (21) also vanishes.

Before presenting the error estimate, we give the following technical result which will be useful in the sequel.

Lemma 2. Let $\boldsymbol{v} \in L^{2}(K)^{2}$ and let $\left(\boldsymbol{u}_{e}^{M}(\boldsymbol{v}), p_{e}^{M}(\boldsymbol{v})\right)$ be the solution of the problem

$$
\begin{array}{rll}
-\nu \Delta \boldsymbol{u}_{e}^{M}(\boldsymbol{v})+\nabla p_{e}^{M}(\boldsymbol{v})=\boldsymbol{v} & , \quad \nabla \cdot \boldsymbol{u}_{e}^{M}(\boldsymbol{v})=0 \quad \text { in } K \\
\boldsymbol{u}_{e}^{M}(\boldsymbol{v})=\mathbf{0} & \text { on } \partial K .
\end{array}
$$

Then, there exists $C>0$, independent of $h$ and $\nu$, such that

$$
\nu\left|\boldsymbol{u}_{e}^{M}(\boldsymbol{v})\right|_{1, K}+\left\|p_{e}^{M}(\boldsymbol{v})\right\|_{0, K} \leq C h_{K}\|\boldsymbol{v}\|_{0, K}
$$

Proof. First, from the weak formulation of (26) we see that $\boldsymbol{u}_{e}^{M}(\boldsymbol{v})$ satisfies

$$
\nu\left|\boldsymbol{u}_{e}^{M}(\boldsymbol{v})\right|_{1, K}^{2} \leq\|\boldsymbol{v}\|_{0, K}\left\|\boldsymbol{u}_{e}^{M}(\boldsymbol{v})\right\|_{0, K}
$$


Now, applying the Poincaré inequality to $\boldsymbol{u}_{e}^{M}(\boldsymbol{v})$ we see that $\left\|\boldsymbol{u}_{e}^{M}(\boldsymbol{v})\right\|_{0, K} \leq C h_{K}\left|\boldsymbol{u}_{e}^{M}(\boldsymbol{v})\right|_{1, K}$, where $C$ does not depend on $h$. Hence, we obtain

$$
\nu\left|\boldsymbol{u}_{e}^{M}(\boldsymbol{v})\right|_{1, K} \leq C h_{K}\|\boldsymbol{v}\|_{0, K} .
$$

Next, from the inf-sup condition (cf. [16]), the weak form of problem (26) and applying once more the Poincaré inequality in $K$ and (28), we see that

$$
\begin{aligned}
\left\|p_{e}^{M}(\boldsymbol{v})\right\|_{0, K} & \leq \beta \sup _{\boldsymbol{w} \in H_{0}^{1}(K)^{2}} \frac{-\left(p_{e}^{M}, \nabla \cdot \boldsymbol{w}\right)_{K}}{|\boldsymbol{w}|_{1, K}}=\beta \sup _{\boldsymbol{w} \in H_{0}^{1}(K)^{2}} \frac{-\nu\left(\nabla \boldsymbol{u}_{e}^{M}(\boldsymbol{v}), \nabla \boldsymbol{w}\right)_{K}+(\boldsymbol{v}, \boldsymbol{w})_{K}}{|\boldsymbol{w}|_{1, K}} \\
& \leq C \beta\left(\nu\left|\boldsymbol{u}_{e}^{M}(\boldsymbol{v})\right|_{1, K}+h_{K}\|\boldsymbol{v}\|_{0, K}\right) \\
& \leq C \beta h_{K}\|\boldsymbol{v}\|_{0, K} .
\end{aligned}
$$

Finally, the inf-sup constant $\beta>0$ may be bounded as follows (cf. [16], Lemma III.3.1)

$$
\beta \leq C\left(\frac{h_{K}}{R}\right)^{2}\left(1+\frac{h_{K}}{R}\right),
$$

where $R$ is the diameter of any ball inscribed in $K$ and $C$ does not depend on $K$ or $h_{K}$. Hence, the desired result arises using the mesh regularity of $\mathcal{T}_{h}$.

Now, we present the main error estimate. For this estimate, we introduce the Clément interpolation operator $\mathcal{C}_{h}$ (cf. $[12,14]$ ), with the obvious extension to vector-valued functions, satisfying

$$
\begin{gathered}
\left\|v-\mathcal{C}_{h}(v)\right\|_{l, K} \leq C h_{K}^{m-l}|v|_{m, \omega_{K}}, \\
\left\|v-\mathcal{C}_{h}(v)\right\|_{0, F} \leq C h_{F}^{m-\frac{1}{2}}|v|_{m, \omega_{F}},
\end{gathered}
$$

for all $v \in H^{m}(\Omega)$, where $0 \leq l \leq m, m=1,2$, and $\omega_{K}:=\cup\left\{K^{\prime}: K \cap K^{\prime} \neq \emptyset\right\}$, $\omega_{F}:=\cup\left\{K^{\prime}: F \cap K^{\prime} \neq \emptyset\right\}$. We recall some standard inequalities needed in the sequel (cf. [14]), namely, the local trace result: there exists $C>0$ such that

$$
\|v\|_{0, F}^{2} \leq C\left(h_{K}^{-1}\|v\|_{0, K}^{2}+h_{K}|v|_{1, K}^{2}\right)
$$

for each edge $F \subset \partial K$ and all $v \in H^{1}(K)$, and the inverse inequality: there exists $C_{I}$ such that

$$
C_{I} h_{K}\|\nabla q\|_{0, K} \leq\|q\|_{0, K},
$$

for all $K \in \mathcal{T}_{h}$ and $q \in P_{1}(K)$. Finally, employing the latter and the generalized Poincaré inequality in each $K$ (see [20]), it is easy to prove the following equivalence:

$$
C_{I} h_{K}|q|_{1, K} \leq\left\|q-\Pi_{K}(q)\right\|_{0, K} \leq \frac{h_{K}}{\pi}|q|_{1, K},
$$


for all $q \in P_{1}(K)$.

Theorem 3. Let us suppose that $(\boldsymbol{u}, p)$, solution of $(2)$, belongs to $H^{2}(\Omega)^{2} \times H^{1}(\Omega)$. Let $\left(\boldsymbol{u}_{1}, p_{1}\right)$ be the solution of (21). Then, there exists $C>0$, independent of $h$ and $\nu$, such that

$$
\begin{aligned}
\left\|\left(\boldsymbol{u}-\boldsymbol{u}_{1}, p-p_{1}\right)\right\|_{h} & \leq C h\left(\sqrt{\nu}\|\boldsymbol{u}\|_{2, \Omega}+\frac{1}{\sqrt{\nu}}|p|_{1, \Omega}\right), \\
\left\|p-p_{1}\right\|_{0, \Omega} & \leq C h\left(\nu\|\boldsymbol{u}\|_{2, \Omega}+|p|_{1, \Omega}\right)
\end{aligned}
$$

Proof. Let $\left(\tilde{\boldsymbol{u}}_{1}, \tilde{p}_{1}\right)=\left(\mathcal{C}_{h}(\boldsymbol{u}), \mathcal{C}_{h}(p)-\Pi_{\Omega}\left(\mathcal{C}_{h}(p)\right)\right),\left(\boldsymbol{w}_{1}, q_{1}\right):=\left(\boldsymbol{u}_{1}-\tilde{\boldsymbol{u}}_{1}, p_{1}-\tilde{p}_{1}\right)$ and $\left(\eta^{\boldsymbol{u}}, \eta^{p}\right):=$ $\left(\boldsymbol{u}-\tilde{\boldsymbol{u}}_{1}, p-\tilde{p}_{1}\right)$. Then,

$$
\left\|\left(\boldsymbol{u}-\boldsymbol{u}_{1}, p-p_{1}\right)\right\|_{h} \leq\left\|\left(\eta^{\boldsymbol{u}}, \eta^{p}\right)\right\|_{h}+\left\|\left(\boldsymbol{w}_{1}, q_{1}\right)\right\|_{h} .
$$

The first term may be bounded using (30)-(31) and the mesh regularity. In fact, we first remark that the interpolation error $\eta^{p}$ also satisfies (30)-(31). Then, using that $\| q-$ $\Pi_{K}(q)\left\|_{0, K} \leq\right\| q \|_{0, K}$ for all $q \in L^{2}(K),\left\|\Pi_{F}(q)\right\|_{0, F} \leq\|q\|_{0, F}$, and the local trace result (32) we obtain

$$
\begin{aligned}
\left\|\left(\eta^{\boldsymbol{u}}, \eta^{p}\right)\right\|_{h}^{2} & \leq \nu\left|\eta^{\boldsymbol{u}}\right|_{1, \Omega}^{2}+\frac{1}{\nu} \sum_{K \in \mathcal{T}_{h}}\left\|\eta^{p}\right\|_{0, K}^{2}+\sum_{F \in \mathcal{E}_{h}} \frac{h_{F}}{12 \nu}\left\|\llbracket \nu \partial_{\boldsymbol{n}} \eta^{\boldsymbol{u}}+\eta^{p} \mathbf{I} \cdot \boldsymbol{n} \rrbracket\right\|_{0, F}^{2} \\
& \leq \nu\left|\eta^{\boldsymbol{u}}\right|_{1, \Omega}^{2}+\frac{1}{\nu}\left\|\eta^{p}\right\|_{0, \Omega}^{2}+C \nu^{-1} \sum_{K \in \mathcal{T}_{h}}\left\|\nu \nabla \eta^{\boldsymbol{u}}+\eta^{p} \mathbf{I}\right\|_{0, K}^{2}+h_{K}^{2}\left|\nu \nabla \boldsymbol{u}+\eta^{p} \mathbf{I}\right|_{1, K}^{2} \\
& \leq C h^{2}\left(\nu|\boldsymbol{u}|_{2, \Omega}^{2}+\frac{1}{\nu}|p|_{1, \Omega}^{2}\right) .
\end{aligned}
$$


For the second term in (37) we use, respectively, Lemma 1, the Cauchy-Schwarz inequality and Lemma 2 to get

$$
\begin{aligned}
\| & \left(\boldsymbol{w}_{1}, q_{1}\right) \|_{h}^{2}=\mathbf{B}\left(\left(\boldsymbol{w}_{1}, q_{1}\right),\left(\boldsymbol{w}_{1}, q_{1}\right)\right) \\
= & \mathbf{B}\left(\left(\eta^{\boldsymbol{u}}, \eta^{p}\right),\left(\boldsymbol{w}_{1}, q_{1}\right)\right) \\
= & \nu\left(\nabla \eta^{\boldsymbol{u}}, \nabla \boldsymbol{w}_{1}\right)_{\Omega}-\left(\eta^{p}, \nabla \cdot \boldsymbol{w}_{1}\right)_{\Omega}+\left(q_{1}, \nabla \cdot \eta^{\boldsymbol{u}}\right)_{\Omega} \\
& +\frac{1}{\nu} \sum_{K \in \mathcal{T}_{h}}\left(\eta^{p}-\Pi_{K}\left(\eta^{p}\right)+p_{e}^{M}(-\nu \Delta \boldsymbol{u}), q_{1}-\Pi_{K}\left(q_{1}\right)\right)_{K} \\
& +\sum_{F \in \mathcal{E}_{h}} \frac{h_{F}}{12 \nu}\left(\Pi_{F}\left(\llbracket \nu \partial_{\boldsymbol{n}} \eta^{\boldsymbol{u}}+\eta^{p} \mathbf{I} \cdot \boldsymbol{n} \rrbracket\right), \Pi_{F}\left(\llbracket \nu \partial_{\boldsymbol{n}} \boldsymbol{w}_{1}+q_{1} \mathbf{I} \cdot \boldsymbol{n} \rrbracket\right)\right)_{F} \\
\leq & \nu\left|\eta^{\boldsymbol{u}}\right|_{1, \Omega}\left|\boldsymbol{w}_{1}\right|_{1, \Omega}+C\left\|\eta^{p}\right\|_{0, \Omega}\left|\boldsymbol{w}_{1}\right|_{1, \Omega}+\left(q_{1}, \nabla \cdot \eta^{\boldsymbol{u}}\right)_{\Omega} \\
& +\frac{1}{\nu} \sum_{K \in \mathcal{T}_{h}}\left(\left\|\eta^{p}-\Pi_{K}\left(\eta^{p}\right)\right\|_{0, K}+\left\|p_{e}^{M}(-\nu \Delta \boldsymbol{u})\right\|_{0, K}\right)\left\|q_{1}-\Pi_{K}\left(q_{1}\right)\right\|_{0, K} \\
& +\sum_{F \in \mathcal{E}_{h}} \frac{h_{F}}{12 \nu}\left\|\Pi_{F}\left(\llbracket \nu \partial_{\boldsymbol{n}} \eta^{\boldsymbol{u}}+\eta^{p} \mathbf{I} \cdot \boldsymbol{n} \rrbracket\right)\right\|_{0, F}\left\|\Pi_{F}\left(\llbracket \nu \partial_{\boldsymbol{n}} \boldsymbol{w}_{1}+q_{1} \mathbf{I} \cdot \boldsymbol{n} \rrbracket\right)\right\|_{0, F} \\
\leq & C\left\{\left\|\left(\eta^{\boldsymbol{u}}, \eta^{p}\right)\right\|_{h}^{2}+\frac{1}{\nu}\left\|\eta^{p}\right\|_{0, \Omega}^{2}+\sum_{K \in \mathcal{T}_{h}} \nu\left\|p_{e}^{M}(\Delta \boldsymbol{u})\right\|_{0, K}^{2}\right\}^{\frac{1}{2}}\left\|\left(\boldsymbol{w}_{1}, q_{1}\right)\right\|_{h}+\left(q_{1}, \nabla \cdot \eta^{\boldsymbol{u}}\right)_{\Omega} \\
\leq & C\left\{\left\|\left(\eta^{\boldsymbol{u}}, \eta^{p}\right)\right\|_{h}^{2}+\frac{h^{2}}{\nu}|p|_{1, \Omega}^{2}+\nu h^{2}|\boldsymbol{u}|_{2, \Omega}^{2}\right\}^{\frac{1}{2}}\left\|\left(\boldsymbol{w}_{1}, q_{1}\right)\right\|_{h}+\left(q_{1}, \nabla \cdot \eta^{\boldsymbol{u}}\right)_{\Omega} \\
\leq & C\left\{\left\|\left(\eta^{\boldsymbol{u}}, \eta^{p}\right)\right\|_{h}^{2}+\frac{h^{2}}{\nu}|p|_{1, \Omega}^{2}+\nu h^{2}|\boldsymbol{u}|_{2, \Omega}^{2}\right\}^{\frac{1}{2}}\left\|\left(\boldsymbol{w}_{1}, q_{1}\right)\right\|_{h} \cdot \\
& \\
(39) &
\end{aligned}
$$

The last step above deserves to be detailed. Integrating by parts and applying (30) we arrive at

$$
\begin{aligned}
& \left(q_{1}, \nabla \cdot \eta^{\boldsymbol{u}}\right)_{\Omega}=-\sum_{K \in \mathcal{T}_{h}}\left(\nabla q_{1}, \eta^{\boldsymbol{u}}\right)_{K}+\sum_{F \in \mathcal{E}_{h}}\left(\llbracket q_{1} \mathbf{I} \cdot \boldsymbol{n} \rrbracket, \eta^{u}\right)_{F} \\
& \leq C \sum_{K \in \mathcal{T}_{h}} h_{K}^{2}\left|q_{1}\right|_{1, K}|\boldsymbol{u}|_{2, \omega_{K}}+\sum_{F \in \mathcal{E}_{h}}\left\|\llbracket q_{1} \mathbf{I} \cdot \boldsymbol{n} \rrbracket-\Pi_{F}\left(\llbracket q_{1} \mathbf{I} \cdot \boldsymbol{n} \rrbracket\right)\right\|_{0, F}\left\|\eta^{u}\right\|_{0, F} \\
& \quad+\sum_{F \in \mathcal{E}_{h}}\left\|\Pi_{F}\left(\llbracket q_{1} \mathbf{I} \cdot \boldsymbol{n} \rrbracket\right)\right\|_{0, F}\left\|\eta^{u}\right\|_{0, F} .
\end{aligned}
$$


Next, we bound term by term. First, applying the equivalence result (34) and the mesh regularity we get

$$
\begin{aligned}
\sum_{K \in \mathcal{T}_{h}} h_{K}^{2}\left|q_{1}\right|_{1, K}|\boldsymbol{u}|_{2, \omega_{K}} & \leq C\left\{\sum_{K \in \mathcal{T}_{h}}\left\|q_{1}-\Pi_{K}\left(q_{1}\right)\right\|_{0, K}^{2}\right\}^{\frac{1}{2}} h|\boldsymbol{u}|_{2, \Omega} \\
& \leq C h \sqrt{\nu}|\boldsymbol{u}|_{2, \Omega}\left\|\left(\boldsymbol{w}_{1}, q_{1}\right)\right\|_{h}
\end{aligned}
$$

For the second term we use the approximation properties of $\Pi_{F}$ (cf. [14]), (31), the local trace result (32), the equivalence result (34) and the mesh regularity to obtain (as in (41))

$$
\begin{aligned}
\sum_{F \in \mathcal{E}_{h}} \| \llbracket q_{1} \mathbf{I} \cdot \boldsymbol{n} \rrbracket & -\Pi_{F}\left(\llbracket q_{1} \mathbf{I} \cdot \boldsymbol{n} \rrbracket\right)\left\|_{0, F}\right\| \eta^{u} \|_{0, F} \leq C \sum_{F \in \mathcal{E}_{h}} h_{F}^{\frac{5}{2}}\left|\llbracket q_{1} \rrbracket\right|_{1, F}|\boldsymbol{u}|_{2, \omega_{F}} \\
& \leq C\left\{\sum_{K \in \mathcal{T}_{h}} h_{K}^{3}\left|q_{1}\right|_{1, \partial K}^{2}\right\}^{\frac{1}{2}}\left\{\sum_{F \in \mathcal{E}_{h}} h_{F}^{2}|\boldsymbol{u}|_{2, \omega_{F}}^{2}\right\}^{\frac{1}{2}} \\
& \leq C\left\{\sum_{K \in \mathcal{T}_{h}} h_{K}^{2}\left|q_{1}\right|_{1, K}^{2}\right\}^{\frac{1}{2}} h|\boldsymbol{u}|_{2, \Omega} \\
& \leq C h \sqrt{\nu}|\boldsymbol{u}|_{2, \Omega}\left\|\left(\boldsymbol{w}_{1}, q_{1}\right)\right\|_{h} .
\end{aligned}
$$

Finally, we treat the third term in (40) by similar arguments to get

$$
\begin{aligned}
& \sum_{F \in \mathcal{E}_{h}}\left\|\Pi_{F}\left(\llbracket q_{1} \mathbf{I} \cdot \boldsymbol{n} \rrbracket\right)\right\|_{0, F}\left\|\eta^{u}\right\|_{0, F} \leq \\
& \leq C \sum_{F \in \mathcal{E}_{h}} h_{F}^{\frac{1}{2}}\left(\left\|\Pi_{F}\left(\llbracket \nu \partial_{\boldsymbol{n}} \boldsymbol{w}_{1}+q_{1} \mathbf{I} \cdot \boldsymbol{n} \rrbracket\right)\right\|_{0, F}+\left\|\Pi_{F}\left(\llbracket \nu \partial_{\boldsymbol{n}} \boldsymbol{w}_{1} \rrbracket\right)\right\|_{0, F}\right) h_{F}|\boldsymbol{u}|_{2, \omega_{F}} \\
& \left.\leq C\left\{\sum_{F \in \mathcal{E}_{h}} h_{F}\left\|\Pi_{F}\left(\llbracket \nu \partial_{\boldsymbol{n}} \boldsymbol{w}_{1}+q_{1} \mathbf{I} \cdot \boldsymbol{n} \rrbracket\right)\right\|_{0, F}^{2}+\sum_{K \in \mathcal{T}_{h}} h_{K}\left\|\nu \partial_{\boldsymbol{n}} \boldsymbol{w}_{1}\right\|_{0, \partial K}^{2}\right)\right\}^{\frac{1}{2}} h|\boldsymbol{u}|_{2, \Omega} \\
& \left.\leq C\left\{\left\|\left(\boldsymbol{w}_{1}, q_{1}\right)\right\|_{h}^{2}+\sum_{K \in \mathcal{T}_{h}} \nu\left|\boldsymbol{w}_{1}\right|_{1, K}^{2}\right)\right\}^{\frac{1}{2}} h \sqrt{\nu}|\boldsymbol{u}|_{2, \Omega} .
\end{aligned}
$$

Gathering contributions (41)-(43), equation (40) becomes

$$
\left(q_{1}, \nabla \cdot \eta^{\boldsymbol{u}}\right)_{\Omega} \leq C \sqrt{\nu} h|\boldsymbol{u}|_{2, \Omega}\left\|\left(\boldsymbol{w}_{1}, q_{1}\right)\right\|_{h},
$$

which was the one used in (39). The first estimate result (35) follows using the interpolation error estimate (38) in (37).

Now we address (36). For that, we use that there exists (cf. [16]) $\boldsymbol{w} \in H_{0}^{1}(\Omega)^{2}$ such that $\nabla \cdot \boldsymbol{w}=p-p_{1}$, and $|\boldsymbol{w}|_{1, \Omega} \leq C\left\|p-p_{1}\right\|_{0, \Omega}$, where $C>0$ depends only on $\Omega$. Let $\boldsymbol{w}_{1}=\mathcal{C}_{h}(\boldsymbol{w})$, 
then using consistency $(25)$ applied to $\left(\boldsymbol{w}_{1}, 0\right)$ and integration by parts

$$
\begin{aligned}
\left\|p-p_{1}\right\|_{0, \Omega}^{2}= & \left(p-p_{1}, \nabla \cdot \boldsymbol{w}\right)_{\Omega} \\
= & \sum_{K \in \mathcal{T}_{h}}\left(p-p_{1}, \nabla \cdot\left(\boldsymbol{w}-\boldsymbol{w}_{1}\right)\right)_{K}+\left(p-p_{1}, \nabla \cdot \boldsymbol{w}_{1}\right)_{\Omega} \\
= & -\sum_{K \in \mathcal{T}_{h}}\left(\nabla\left(p-p_{1}\right), \boldsymbol{w}-\boldsymbol{w}_{1}\right)_{K}+\sum_{F \in \mathcal{E}_{h}}\left(\llbracket\left(p-p_{1}\right) \mathbf{I} \cdot \boldsymbol{n} \rrbracket, \boldsymbol{w}-\boldsymbol{w}_{1}\right)_{F} \\
& +\nu\left(\nabla\left(\boldsymbol{u}-\boldsymbol{u}_{1}\right), \nabla \boldsymbol{w}_{1}\right)_{\Omega} \\
& +\sum_{F \in \mathcal{E}_{h}} \frac{h_{F}}{12 \nu}\left(\Pi_{F}\left(\llbracket \nu \partial_{\boldsymbol{n}}\left(\boldsymbol{u}-\boldsymbol{u}_{1}\right)+\left(p-p_{1}\right) \mathbf{I} \cdot \boldsymbol{n} \rrbracket\right), \Pi_{F}\left(\llbracket \nu \partial_{\boldsymbol{n}} \boldsymbol{w}_{1} \rrbracket\right)\right)_{F} .
\end{aligned}
$$

The next step is to bound each one of the terms above. To this end, we use (30) to proceed as in (41) to estimate the first term

$$
\begin{aligned}
& \sum_{K \in \mathcal{T}_{h}}\left(\nabla\left(p-p_{1}\right), \boldsymbol{w}-\boldsymbol{w}_{1}\right)_{K} \leq C \sum_{K \in \mathcal{T}_{h}} h_{K}\left|p-p_{1}\right|_{1, K}|\boldsymbol{w}|_{1, \omega_{K}} \\
& \leq C\left\{\sum_{K \in \mathcal{T}_{h}} h_{K}^{2}|p|_{1, K}^{2}+h_{K}^{2}\left|p_{1}-\Pi_{K}\left(p_{1}\right)\right|_{1, K}^{2}\right\}^{\frac{1}{2}}|\boldsymbol{w}|_{1, \Omega} \\
& \leq C\left\{h^{2}|p|_{1, \Omega}^{2}+\sum_{K \in \mathcal{T}_{h}}\left\|p_{1}-\Pi_{K}\left(p_{1}\right)\right\|_{0, K}^{2}\right\}^{\frac{1}{2}}|\boldsymbol{w}|_{1, \Omega} \\
& \leq C\left\{h^{2}|p|_{1, \Omega}^{2}+\sum_{K \in \mathcal{T}_{h}}\left\|p-p_{1}-\Pi_{K}\left(p-p_{1}\right)\right\|_{0, K}^{2}+\left\|p-\Pi_{K}(p)\right\|_{0, K}^{2}\right\}^{\frac{1}{2}}|\boldsymbol{w}|_{1, \Omega} \\
& \leq C\left\{\frac{h^{2}}{\nu}|p|_{1, \Omega}^{2}+\left\|\left(\boldsymbol{u}-\boldsymbol{u}_{1}, p-p_{1}\right)\right\|_{h}^{2}\right\}^{\frac{1}{2}} \sqrt{\nu}\left\|p-p_{1}\right\|_{0, \Omega} .
\end{aligned}
$$

Using the Cauchy-Schwarz inequality combined with (31) and the mesh regularity we estimate the the second term by

$$
\begin{aligned}
& \sum_{F \in \mathcal{E}_{h}}\left(\llbracket\left(p-p_{1}\right) \mathbf{I} \cdot \boldsymbol{n} \rrbracket, \boldsymbol{w}-\boldsymbol{w}_{1}\right)_{F} \\
& \quad \leq \sum_{F \in \mathcal{E}_{h}}\left(\left\|\llbracket\left(p-p_{1}\right) \mathbf{I} \cdot \boldsymbol{n} \rrbracket-\Pi_{F}\left(\llbracket\left(p-p_{1}\right) \mathbf{I} \cdot \boldsymbol{n} \rrbracket\right)\right\|_{0, F}+\left\|\Pi_{F}\left(\llbracket\left(p-p_{1}\right) \mathbf{I} \cdot \boldsymbol{n} \rrbracket\right)\right\|_{0, F}\right)\left\|\boldsymbol{w}-\boldsymbol{w}_{1}\right\|_{0, F}
\end{aligned}
$$

$$
\leq C\left\{\sum_{F \in \mathcal{E}_{h}} h_{F}\left(\left\|\llbracket p-p_{1} \rrbracket-\Pi_{F}\left(\llbracket p-p_{1} \rrbracket\right)\right\|_{0, F}^{2}+\left\|\Pi_{F}\left(\llbracket\left(p-p_{1}\right) \mathbf{I} \cdot \boldsymbol{n} \rrbracket\right)\right\|_{0, F}^{2}\right)\right\}^{\frac{1}{2}}|\boldsymbol{w}|_{1, \Omega} .
$$


Now, to bound (47), recalling that the jump function on $F=K \cap K^{\prime} \in \mathcal{E}_{h}$ satisfies $\Pi_{F}\left(\llbracket p-p_{1} \rrbracket\right)=\Pi_{F}\left(\left.\left(p-p_{1}\right)\right|_{K}\right)-\Pi_{F}\left(\left.\left(p-p_{1}\right)\right|_{K^{\prime}}\right)$, and using the mesh regularity we obtain (as in (46))

$$
\begin{aligned}
\sum_{F \in \mathcal{E}_{h}} h_{F} \| \llbracket p-p_{1} \rrbracket & -\Pi_{F}\left(\llbracket p-p_{1} \rrbracket\right)\left\|_{0, F}^{2} \leq C \sum_{K \in \mathcal{T}_{h}} \sum_{F \subseteq \partial K \cap \Omega} h_{K}\right\| p-p_{1}-\Pi_{F}\left(p-p_{1}\right) \|_{0, F}^{2} \\
& \leq C \sum_{K \in \mathcal{T}_{h}} \sum_{F \subseteq \partial K \cap \Omega} h_{K}\left\|p-p_{1}-\Pi_{K}\left(p-p_{1}\right)\right\|_{0, F}^{2} \\
& \leq C \sum_{K \in \mathcal{T}_{h}}\left\|p-p_{1}-\Pi_{K}\left(p-p_{1}\right)\right\|_{0, K}^{2}+h_{K}^{2}\left|p-p_{1}\right|_{1, K}^{2} \\
& \leq C \nu\left(\left\|\left(\boldsymbol{u}-\boldsymbol{u}_{1}, p-p_{1}\right)\right\|_{h}^{2}+\frac{h^{2}}{\nu}|p|_{1, \Omega}^{2}\right) .
\end{aligned}
$$

Similarly, we prove that

$$
\begin{aligned}
& \sum_{F \in \mathcal{E}_{h}} h_{F}\left\|\Pi_{F}\left(\llbracket\left(p-p_{1}\right) \mathbf{I} \cdot \boldsymbol{n} \rrbracket\right)\right\|_{0, F}^{2} \\
& \leq \sum_{F \in \mathcal{E}_{h}} h_{F}\left\|\Pi_{F}\left(\llbracket \nu \partial_{\boldsymbol{n}}\left(\boldsymbol{u}-\boldsymbol{u}_{1}\right)+\left(p-p_{1}\right) \mathbf{I} \cdot \boldsymbol{n} \rrbracket\right)\right\|_{0, F}^{2}+\left\|\Pi_{F}\left(\llbracket \nu \partial_{\boldsymbol{n}}\left(\boldsymbol{u}-\boldsymbol{u}_{1}\right) \rrbracket\right)\right\|_{0, F}^{2} \\
& \quad \leq C \nu\left(\left\|\left(\boldsymbol{u}-\boldsymbol{u}_{1}, p-p_{1}\right)\right\|_{h}^{2}+\nu h^{2}|\boldsymbol{u}|_{2, \Omega}^{2}\right) .
\end{aligned}
$$

Hence, (47) becomes

$$
\begin{aligned}
& \sum_{F \in \mathcal{E}_{h}}\left(\llbracket\left(p-p_{1}\right) \mathbf{I} \cdot \boldsymbol{n} \rrbracket, \boldsymbol{w}-\boldsymbol{w}_{1}\right)_{F} \\
& \quad \leq C \sqrt{\nu}\left(\left\|\left(\boldsymbol{u}-\boldsymbol{u}_{1}, p-p_{1}\right)\right\|_{h}^{2}+\nu h^{2}|\boldsymbol{u}|_{2, \Omega}^{2}+\frac{h^{2}}{\nu}|p|_{1, \Omega}^{2}\right)^{\frac{1}{2}}\left\|p-p_{1}\right\|_{0, \Omega} .
\end{aligned}
$$

The last term in (45) is tackled using Cauchy-Schwarz's inequality leading to

$$
\begin{aligned}
\nu\left(\nabla\left(\boldsymbol{u}-\boldsymbol{u}_{1}\right), \nabla \boldsymbol{w}_{1}\right)_{\Omega} & +\sum_{F \in \mathcal{E}_{h}} \frac{h_{F}}{12 \nu}\left(\Pi_{F}\left(\llbracket \nu \partial_{\boldsymbol{n}}\left(\boldsymbol{u}-\boldsymbol{u}_{1}\right)+\left(p-p_{1}\right) \mathbf{I} \cdot \boldsymbol{n} \rrbracket\right), \Pi_{F}\left(\llbracket \nu \partial_{\boldsymbol{n}} \boldsymbol{w}_{1} \rrbracket\right)\right)_{F} \\
& \leq C\left\|\left(\boldsymbol{u}-\boldsymbol{u}_{1}, p-p_{1}\right)\right\|_{h} \sqrt{\nu}\left\|p-p_{1}\right\|_{0, \Omega} .
\end{aligned}
$$

Finally, gathering contributions (46), (50) and (51) we end up with

$$
\left\|p-p_{1}\right\|_{0, \Omega}^{2} \leq C \sqrt{\nu}\left(\left\|\left(\boldsymbol{u}-\boldsymbol{u}_{1}, p-p_{1}\right)\right\|_{h}^{2}+\frac{h^{2}}{\nu}|p|_{1, \Omega}^{2}+\nu h^{2}|\boldsymbol{u}|_{2, \Omega}\right)^{\frac{1}{2}}\left\|p-p_{1}\right\|_{0, \Omega},
$$

and the result follows dividing by $\left\|p-p_{1}\right\|_{0, \Omega}$ and using (35).

We end this section proving an optimal error estimate for $\left\|\boldsymbol{u}-\boldsymbol{u}_{1}\right\|_{0, \Omega}$. 
Lemma 4. Let us suppose that $\Omega$ is a convex polygon. Then, there exists $C>0$, independent of $h$ and $\nu$, such that

$$
\left\|\boldsymbol{u}-\boldsymbol{u}_{1}\right\|_{0, \Omega} \leq C h^{2}\left(|\boldsymbol{u}|_{2, \Omega}+\frac{1}{\nu}|p|_{1, \Omega}\right) .
$$

Proof. We consider the dual problem

$$
\begin{gathered}
-\nu \Delta \boldsymbol{\psi}-\nabla \xi=\boldsymbol{u}-\boldsymbol{u}_{1} \quad, \quad \nabla \cdot \boldsymbol{\psi}=0 \quad \text { in } \Omega \\
\boldsymbol{\psi}=\mathbf{0} \quad \text { on } \partial \Omega .
\end{gathered}
$$

Since $\Omega$ is a convex polygon, then $(\boldsymbol{\psi}, \xi) \in H^{2}(\Omega)^{2} \times H^{1}(\Omega)$, and the following estimate holds

$$
\nu\|\boldsymbol{\psi}\|_{2, \Omega}+\|\xi\|_{1, \Omega} \leq C\left\|\boldsymbol{u}-\boldsymbol{u}_{1}\right\|_{0, \Omega} .
$$

Now, multiplying the first equation in (54) by $\boldsymbol{u}-\boldsymbol{u}_{1}$, the second one by $-\left(p-p_{1}\right)$, integrating by parts, using the definition of $\mathbf{B}(.,$.$) and the fact that \llbracket \partial_{\boldsymbol{n}} \boldsymbol{\psi} \rrbracket=\mathbf{0}$ and $\llbracket \xi \rrbracket=0$ we obtain

$$
\begin{aligned}
\| \boldsymbol{u} & -\boldsymbol{u}_{1} \|_{0, \Omega}^{2}=\nu\left(\nabla\left(\boldsymbol{u}-\boldsymbol{u}_{1}\right), \nabla \boldsymbol{\psi}\right)_{\Omega}-\left(p-p_{1}, \nabla \cdot \boldsymbol{\psi}\right)_{\Omega}+\left(\xi, \nabla \cdot\left(\boldsymbol{u}-\boldsymbol{u}_{1}\right)\right)_{\Omega} \\
& =\mathbf{B}\left(\left(\boldsymbol{u}-\boldsymbol{u}_{1}, p-p_{1}\right),(\boldsymbol{\psi}, \xi)\right)-\frac{1}{\nu} \sum_{K \in \mathcal{T}_{h}}\left(p-p_{1}-\Pi_{K}\left(p-p_{1}\right)+p_{e}^{M}(-\nu \Delta \boldsymbol{u}), \xi-\Pi_{K}(\xi)\right)_{K} .
\end{aligned}
$$

Now, we define $\psi_{1}:=\mathcal{C}_{h}(\boldsymbol{\psi})$ and $\xi_{1}:=\mathcal{C}_{h}(\xi)-\Pi_{\Omega}\left(\mathcal{C}_{h}(\xi)\right)$. Then, using the Cauchy-Schwarz inequality, (32) and (30), we arrive at

$$
\begin{aligned}
\left\|\boldsymbol{u}-\boldsymbol{u}_{1}\right\|_{0, \Omega}^{2}=\mathbf{B}\left(\left(\boldsymbol{u}-\boldsymbol{u}_{1}, p-p_{1}\right),\left(\boldsymbol{\psi}-\boldsymbol{\psi}_{1}, \xi-\xi_{1}\right)\right) \\
\quad-\frac{1}{\nu} \sum_{K \in \mathcal{T}_{h}}\left(p-p_{1}-\Pi_{K}\left(p-p_{1}\right)+p_{e}^{M}(-\nu \Delta \boldsymbol{u}), \xi-\Pi_{K}(\xi)\right)_{K} \\
\leq C\left\{\left\|\left(\boldsymbol{u}-\boldsymbol{u}_{1}, p-p_{1}\right)\right\|_{h}^{2}+\frac{1}{\nu}\left\|p-p_{1}\right\|_{0, \Omega}^{2}+\frac{1}{\nu} \sum_{K \in \mathcal{T}_{h}} \nu^{2}\left\|p_{e}^{M}(\Delta \boldsymbol{u})\right\|_{0, K}^{2}\right\}^{\frac{1}{2}} \\
\quad\left\{\left\|\left(\boldsymbol{\psi}-\boldsymbol{\psi}_{1}, \xi-\xi_{1}\right)\right\|_{h}^{2}+\frac{1}{\nu}\left\|\xi-\xi_{1}\right\|_{0, \Omega}^{2}+\frac{1}{\nu} \sum_{K \in \mathcal{T}_{h}}\left\|\xi-\Pi_{K}(\xi)\right\|_{0, K}^{2}\right\}^{\frac{1}{2}},
\end{aligned}
$$

and the result follows using (38),(30), the approximation properties of $\Pi_{K}$ (cf. [14]), (55),(35), (36), Lemma 2 and dividing by $\left\|\boldsymbol{u}-\boldsymbol{u}_{1}\right\|_{0, \Omega}$.

3.5. Recovering a locally mass-conservative velocity field. As we mentioned in the introduction, the local mass conservation property is usually overlooked in the numerical solution of Stokes flow, but this property is of capital importance when solving a coupled problem such as heat transfer. This is why we now propose two ways to build a locally mass conservative velocity field, having the same convergence properties of $\boldsymbol{u}_{1}$ (we state at this 
point that the results presented in this section may be directly applied to the $\mathbb{P}_{1} / \mathbb{P}_{0}$ method from §3.1). First, as in [4] we can consider $\boldsymbol{u}_{e}^{D}$ given as the solution of (10) (with $\alpha=1$ ) and get an enriched velocity field $\tilde{\boldsymbol{u}}_{h}:=\boldsymbol{u}_{1}+\boldsymbol{u}_{e}^{D}$, satisfying the following result.

Lemma 5. Let $\tilde{\boldsymbol{u}}_{h}:=\boldsymbol{u}_{1}+\boldsymbol{u}_{e}^{D}$, where $\boldsymbol{u}_{1}$ and $\boldsymbol{u}_{e}^{D}$ are the solutions of (21) and (10), respectively. Then,

$$
\nabla \cdot \tilde{\boldsymbol{u}}_{h}=0 \quad \forall K \in \mathcal{T}_{h}
$$

Moreover, if $(\boldsymbol{u}, p) \in H^{2}(\Omega)^{2} \times H^{1}(\Omega)$ is the solution of (2), then there exists a constant $C>0$, independent of $h$ and $\nu$, such that

$$
\left|\boldsymbol{u}-\tilde{\boldsymbol{u}}_{h}\right|_{1, \Omega} \leq C h\left(\|\boldsymbol{u}\|_{2, \Omega}+\frac{1}{\nu}|p|_{1, \Omega}\right)
$$

and, assuming $\Omega$ a convex polygon, the following estimate holds

$$
\left\|\boldsymbol{u}-\tilde{\boldsymbol{u}}_{h}\right\|_{0, \Omega} \leq C h^{2}\left(\|\boldsymbol{u}\|_{2, \Omega}+\frac{1}{\nu}|p|_{1, \Omega}\right) .
$$

Proof. Let $K^{\prime} \in \mathcal{T}_{h}$ be a fixed element and $K$ any other element of the triangulation, and let us define the function $q_{1} \in Q_{h}^{1}$ as follows: $q_{1}=1$ in $K^{\prime}, q_{1}=-\frac{\left|K^{\prime}\right|}{|K|}$ in $K$, and zero everywhere else. Then, using $\left(\mathbf{0}, q_{1}\right)$ as test function in the definition of the method (cf. (21)) we get

$$
\left(q_{1}, \nabla \cdot \boldsymbol{u}_{1}\right)_{K \cup K^{\prime}}+\sum_{F \in \mathcal{E}_{h}} \frac{h_{F}}{12 \nu}\left(\Pi_{F}\left(\llbracket \nu \partial_{\boldsymbol{n}} \boldsymbol{u}_{1}+p_{1} \mathbf{I} \cdot \boldsymbol{n} \rrbracket\right), \llbracket q_{1} \mathbf{I} \cdot \boldsymbol{n} \rrbracket\right)_{F}=0,
$$

and then, integrating by parts and using (10) we obtain

$$
\left(1, \nabla \cdot\left(\boldsymbol{u}_{1}+\boldsymbol{u}_{e}^{D}\right)\right)_{K}=\frac{|K|}{\left|K^{\prime}\right|}\left(1, \nabla \cdot\left(\boldsymbol{u}_{1}+\boldsymbol{u}_{e}^{D}\right)\right)_{K^{\prime}}
$$

Now, since $\left(\boldsymbol{u}_{1}+\boldsymbol{u}_{e}^{D}\right) \cdot \boldsymbol{n}=0$ on $\partial \Omega$ we have

$$
0=\left(1, \nabla \cdot\left(\boldsymbol{u}_{1}+\boldsymbol{u}_{e}^{D}\right)\right)_{\Omega}=\left\{\sum_{K \in \mathcal{T}_{h}} \frac{|K|}{\left|K^{\prime}\right|}\right\}\left(1, \nabla \cdot\left(\boldsymbol{u}_{1}+\boldsymbol{u}_{e}^{D}\right)\right)_{K^{\prime}},
$$

leading to $\left(1, \nabla \cdot\left(\boldsymbol{u}_{1}+\boldsymbol{u}_{e}^{D}\right)\right)_{K^{\prime}}=0$. The result follows using that $\nabla \cdot\left(\boldsymbol{u}_{1}+\boldsymbol{u}_{e}^{D}\right) \in \mathbb{R}$ in each element of $\mathcal{T}_{h}$.

The proof of the error estimate reduces to prove a bound for $\left|\boldsymbol{u}_{e}^{D}\right|_{1, K}$ in each $K \in \mathcal{T}_{h}$. To do this, we multiply (10) by $\boldsymbol{u}_{e}^{D}$, integrate by parts, use that $\|\boldsymbol{v} \cdot \boldsymbol{n}\|_{-\frac{1}{2}, \partial K} \leq\|\boldsymbol{v}\|_{d i v, K}$ (cf. 
$[18])$, and (10) to obtain that

$$
\begin{aligned}
\nu\left|\boldsymbol{u}_{e}^{D}\right|_{1, K}^{2} & =-\left(\boldsymbol{u}_{e}^{D},-\nu \partial_{\boldsymbol{n}} \boldsymbol{u}_{e}^{D}+p_{e}^{D} \mathbf{I} \cdot \boldsymbol{n}\right)_{\partial K} \\
& \leq\left\|\boldsymbol{u}_{e}^{D}\right\|_{\frac{1}{2}, \partial K}\left\|-\nu \partial_{\boldsymbol{n}} \boldsymbol{u}_{e}^{D}+p_{e}^{D} \mathbf{I} \cdot \boldsymbol{n}\right\|_{-\frac{1}{2}, \partial K} \\
& \leq\left\|\boldsymbol{g}_{e}\right\|_{\frac{1}{2}, \partial K}\left\{\left\|-\nu \nabla \boldsymbol{u}_{e}^{D}+p_{e}^{D} \mathbf{I}\right\|_{0, K}^{2}+\left\|\nabla \cdot\left(-\nu \nabla \boldsymbol{u}_{e}^{D}+p_{e}^{D} \mathbf{I}\right)\right\|_{0, K}^{2}\right\}^{\frac{1}{2}} \\
& \leq C\left\|\boldsymbol{g}_{e}\right\|_{\frac{1}{2}, \partial K}\left(\nu\left|\boldsymbol{u}_{e}^{D}\right|_{1, K}+\left\|p_{e}^{D}\right\|_{0, K}\right)
\end{aligned}
$$

Next, following analogous steps as in the proof of Lemma 2, we obtain that there exists a constant $C>0$, independent of $h_{K}$, such that

$$
\left\|p_{e}^{D}\right\|_{0, K} \leq C \nu\left|\boldsymbol{u}_{e}^{D}\right|_{1, K}
$$

and then, there exists $C>0$, independent of $h$, such that

$$
\left|\boldsymbol{u}_{e}^{D}\right|_{1, K} \leq C\left\|\boldsymbol{g}_{e}\right\|_{\frac{1}{2}, \partial K}
$$

and since $\boldsymbol{g}_{e}$ is a polynomial of degree two in each edge, we use an inverse estimate (cf. [14]) to see that $\left\|\boldsymbol{g}_{e}\right\|_{\frac{1}{2}, \partial K} \leq C h_{K}^{-\frac{1}{2}}\left\|\boldsymbol{g}_{e}\right\|_{0, \partial K}$, leading to

$$
\left|\boldsymbol{u}_{e}^{D}\right|_{1, K} \leq C h_{K}^{-\frac{1}{2}}\left\|\boldsymbol{g}_{e}\right\|_{0, \partial K}
$$

In addition, since $\left.\boldsymbol{g}_{e}\right|_{F}=g_{F} \mathbf{I} \cdot \Pi_{F}\left(\llbracket \nu \partial_{\boldsymbol{n}} \boldsymbol{u}_{1}+\left.p_{1} \mathbf{I} \cdot \boldsymbol{n} \rrbracket\right|_{F}\right)$, where $g_{F}$ satisfies $g_{F} \leq C \nu^{-1} h_{F}$ in $F$, then

$$
\left\|\boldsymbol{g}_{e}\right\|_{0, F}^{2} \leq \int_{F} g_{F}^{2}\left|\Pi_{F}\left(\llbracket \nu \partial_{\boldsymbol{n}} \boldsymbol{u}_{1}+p_{1} \mathbf{I} \cdot \boldsymbol{n} \rrbracket\right)\right|^{2} \leq \frac{C h_{F}^{2}}{\nu^{2}}\left\|\Pi_{F}\left(\llbracket \nu \partial_{\boldsymbol{n}} \boldsymbol{u}_{1}+p_{1} \mathbf{I} \cdot \boldsymbol{n} \rrbracket\right)\right\|_{0, F}^{2}
$$

Hence, using (65), (64) leads to

$$
\begin{aligned}
\left|\boldsymbol{u}_{e}^{D}\right|_{1, K} & \leq C h_{K}^{-\frac{1}{2}}\left\{\sum_{F \subset \partial K \cap \Omega} \frac{h_{F}^{2}}{\nu^{2}}\left\|\Pi_{F}\left(\llbracket \nu \partial_{\boldsymbol{n}} \boldsymbol{u}_{1}+p_{1} \mathbf{I} \cdot \boldsymbol{n} \rrbracket\right)\right\|_{0, F}^{2}\right\}^{\frac{1}{2}} \\
& \leq \frac{C}{\sqrt{\nu}}\left\{\sum_{F \subset \partial K \cap \Omega} \frac{h_{F}}{\nu}\left\|\Pi_{F}\left(\llbracket \nu \partial_{\boldsymbol{n}} \boldsymbol{u}_{1}+p_{1} \mathbf{I} \cdot \boldsymbol{n} \rrbracket\right)\right\|_{0, F}^{2}\right\}^{\frac{1}{2}} .
\end{aligned}
$$


Then, squaring, summing over all the triangles of $\mathcal{T}_{h}$, using that $\llbracket \partial_{\boldsymbol{n}} \boldsymbol{u} \rrbracket=\mathbf{0}$ and $\llbracket p \rrbracket=0$ and (35) we obtain

$$
\begin{aligned}
\left|\boldsymbol{u}_{e}^{D}\right|_{1, K} & \leq C \nu^{-\frac{1}{2}}\left\{\sum_{F \in \mathcal{E}_{h}} \frac{h_{F}}{12 \nu}\left\|\Pi_{F}\left(\llbracket \nu \partial_{\boldsymbol{n}}\left(\boldsymbol{u}-\boldsymbol{u}_{1}\right)+\left(p-p_{1}\right) \mathbf{I} \cdot \boldsymbol{n} \rrbracket\right)\right\|_{0, F}^{2}\right\}^{\frac{1}{2}} \\
& \leq C \nu^{-\frac{1}{2}}\left\|\left(\boldsymbol{u}-\boldsymbol{u}_{1}, p-p_{1}\right)\right\|_{h} \\
& \leq C h\left(|\boldsymbol{u}|_{2, \Omega}+\frac{1}{\nu}|p|_{1, \Omega}\right)
\end{aligned}
$$

and (57) follows. To prove (58) we first remark that, for a function $v \in H^{1}(K)$, a standard scaling argument leads to the following generalized Poincaré inequality

$$
\|v\|_{0, K} \leq C h_{K}\left(|v|_{1, K}+\sum_{F \subset \partial K} h_{F}^{-\frac{1}{2}}\|v\|_{0, F}\right),
$$

where $C>0$ does not depend on $h_{K}$. Applying this result to $\boldsymbol{u}_{e}^{D}$ and using (65) we obtain

$$
\begin{aligned}
\left\|\boldsymbol{u}_{e}^{D}\right\|_{0, K} & \leq C h_{K}\left(\left|\boldsymbol{u}_{e}^{D}\right|_{1, K}+\sum_{F \subset \partial K \cap \Omega} h_{F}^{-\frac{1}{2}}\left\|\boldsymbol{g}_{e}\right\|_{0, F}\right) \\
& \leq C h_{K}\left(\left|\boldsymbol{u}_{e}^{D}\right|_{1, K}+\sum_{F \subset \partial K \cap \Omega} \frac{h_{F}^{\frac{1}{2}}}{\nu}\left\|\Pi_{F}\left(\llbracket \nu \partial_{\boldsymbol{n}} \boldsymbol{u}_{1}+p_{1} \mathbf{I} \cdot \boldsymbol{n} \rrbracket\right)\right\|_{0, F}\right),
\end{aligned}
$$

and then (58) follows using the same arguments as for (67).

This first approach has the advantage of providing a continuous velocity field, with the same nodal values as $\boldsymbol{u}_{1}$, but at the cost of solving the local problem (10) as a post-process after the solution. To avoid this local problem solutions, we define in each $K \in \mathcal{T}_{h}$ (see [5] for a related idea)

$$
\boldsymbol{u}_{n c}=\sum_{F \subset \partial K \cap \Omega} \frac{h_{F}}{12 \nu} \Pi_{F}\left(\llbracket \nu \partial_{\boldsymbol{n}} \boldsymbol{u}_{1}+\left.p_{1} \mathbf{I} \cdot \boldsymbol{n} \rrbracket\right|_{F}\right) \boldsymbol{\varphi}_{F}
$$

where $\boldsymbol{\varphi}_{F}$ is the basis function of the lowest order Raviart-Thomas space given by

$$
\boldsymbol{\varphi}_{F}(\mathbf{x})=\frac{h_{F}}{2|K|}\left(\mathbf{x}-\mathbf{x}_{F}\right)
$$

and $\mathbf{x}_{F}$ is the node opposite to the edge $F$. Using this function, we can build the nonconforming velocity field $\boldsymbol{u}_{h}:=\boldsymbol{u}_{1}+\boldsymbol{u}_{n c}$ which satisfies the local conservation of mass property and shares the same convergence properties as $\boldsymbol{u}_{1}$. This is stated in the next result. 
Lemma 6. Let $\boldsymbol{u}_{1}$ be the solution of (21) and $\boldsymbol{u}_{n c}$ given in (70), respectively. The velocity field $\overline{\boldsymbol{u}}_{h}:=\boldsymbol{u}_{1}+\boldsymbol{u}_{n c}$ satisfies

$$
\nabla \cdot \overline{\boldsymbol{u}}_{h}=0 \quad \text { in each } K \in \mathcal{T}_{h} .
$$

Furthermore, being $(\boldsymbol{u}, p) \in H^{2}(\Omega)^{2} \times H^{1}(\Omega)$ the solution of (2), the following error estimate is valid

$$
\left\{\sum_{K \in \mathcal{T}_{h}}\left|\boldsymbol{u}-\overline{\boldsymbol{u}}_{h}\right|_{1, K}^{2}\right\}^{\frac{1}{2}} \leq C h\left(\|\boldsymbol{u}\|_{2, \Omega}+\frac{1}{\nu}|p|_{1, \Omega}\right),
$$

where $C>0$ neither depend on $h$ nor $\nu$. In the case that $\Omega$ is a convex polygon, the following estimate holds

$$
\left\|\boldsymbol{u}-\overline{\boldsymbol{u}}_{h}\right\|_{0, \Omega} \leq C h^{2}\left(\|\boldsymbol{u}\|_{2, \Omega}+\frac{1}{\nu}|p|_{1, \Omega}\right) .
$$

Proof. The local conservation of mass arises as in the previous Lemma. For the error estimate we first remark that, due to the mesh regularity and the definition of $\boldsymbol{\varphi}_{F}$ (cf. (71)), $\left|\boldsymbol{\varphi}_{F}\right|_{1, K} \leq$ $C$, and then using the Cauchy-Schwarz inequality and $\llbracket \partial_{\boldsymbol{n}} \boldsymbol{u} \rrbracket=\mathbf{0}, \llbracket p \rrbracket=0$, we arrive at

$$
\begin{aligned}
& \left|\boldsymbol{u}_{n c}\right|_{1, K} \leq C \sum_{F \subset \partial K} \frac{h_{F}}{\nu}\left|\Pi_{F}\left(\llbracket \nu \partial_{\boldsymbol{n}} \boldsymbol{u}_{1}+p_{1} \mathbf{I} \cdot \boldsymbol{n} \rrbracket\right)\right|_{F} \mid \\
& \quad=\frac{C}{\nu} \sum_{F \subset \partial K} \int_{F}\left|\Pi_{F}\left(\llbracket \nu \partial_{\boldsymbol{n}} \boldsymbol{u}_{1}+p_{1} \mathbf{I} \cdot \boldsymbol{n} \rrbracket\right)\right| \\
& \quad \leq \frac{C}{\nu} \sum_{F \subset \partial K} h_{F}^{\frac{1}{2}}\left\|\Pi_{F}\left(\llbracket \nu \partial_{\boldsymbol{n}} \boldsymbol{u}_{1}+p_{1} \mathbf{I} \cdot \boldsymbol{n} \rrbracket\right)\right\|_{0, F} \\
& \quad \leq \frac{C}{\nu} \sum_{F \subset \partial K} h_{F}^{\frac{1}{2}}\left\|\Pi_{F}\left(\llbracket \nu \partial_{\boldsymbol{n}}\left(\boldsymbol{u}-\boldsymbol{u}_{1}\right)+\left(p-p_{1}\right) \mathbf{I} \cdot \boldsymbol{n} \rrbracket\right)\right\|_{0, F},
\end{aligned}
$$

and (72) follows as in (67). Finally, the estimate for $\left\|\boldsymbol{u}-\overline{\boldsymbol{u}}_{h}\right\|_{0, \Omega}$ is done following the exact same steps and using that $\left\|\boldsymbol{\varphi}_{F}\right\|_{0, K} \leq C h_{K}$.

\section{A NUMERICAL EXPERIMENT}

Before heading to the numerical experiments, we make a short remark on the final implementation of the method. If the function $\boldsymbol{f}$ is not a piecewise constant, then we must be able to solve problem (8) before implementing the method, thus, leading to a two-level method. If we want to avoid this, we can approximate $\boldsymbol{f}$ by $\Pi_{K}(\boldsymbol{f})$ in each element and just use the analytical solution from (11). Now, this approximation introduces a consistency error, but, we can prove that it is of a smaller order. In fact, let us suppose that $\boldsymbol{f} \in H^{1}(\Omega)^{2}$ and let us denote by $\left(\tilde{\boldsymbol{u}}_{1}, \tilde{p}_{1}\right)$ the solution using the method with $p_{e}^{M}\left(\Pi_{K}(\boldsymbol{f})\right)$ on the right-hand side. 
Then, using the Lemma 2, Cauchy-Schwarz's inequality and the approximation properties of $\Pi_{K}$ (cf. [14]), we obtain

$$
\begin{aligned}
\|\left(\boldsymbol{u}_{1}\right. & \left.-\tilde{\boldsymbol{u}}_{1}, p_{1}-\tilde{p}_{1}\right) \|_{h}^{2}=\mathbf{B}\left(\left(\boldsymbol{u}_{1}-\tilde{\boldsymbol{u}}_{1}, p_{1}-\tilde{p}_{1}\right),\left(\boldsymbol{u}_{1}-\tilde{\boldsymbol{u}}_{1}, p_{1}-\tilde{p}_{1}\right)\right) \\
& =\frac{1}{\nu} \sum_{K \in \mathcal{T}_{h}}\left(p_{e}^{M}(\boldsymbol{f})-p_{e}^{M}\left(\Pi_{K}(\boldsymbol{f})\right), p_{1}-\tilde{p}_{1}-\Pi_{K}\left(p_{1}-\tilde{p}_{1}\right)\right)_{K} \\
& \leq \frac{1}{\nu} \sum_{K \in \mathcal{T}_{h}}\left\|p_{e}^{M}(\boldsymbol{f})-p_{e}^{M}\left(\Pi_{K}(\boldsymbol{f})\right)\right\|_{0, K}\left\|p_{1}-\tilde{p}_{1}-\Pi_{K}\left(p_{1}-\tilde{p}_{1}\right)\right\|_{0, K} \\
& \leq \frac{1}{\sqrt{\nu}}\left\{\sum_{K \in \mathcal{T}_{h}}\left\|p_{e}^{M}\left(\boldsymbol{f}-\Pi_{K}(\boldsymbol{f})\right)\right\|_{0, K}^{2}\right\}^{\frac{1}{2}}\left\|\left(\boldsymbol{u}_{1}-\tilde{\boldsymbol{u}}_{1}, p_{1}-\tilde{p}_{1}\right)\right\|_{h} \\
& \leq \frac{1}{\sqrt{\nu}}\left\{\sum_{K \in \mathcal{T}_{h}} C h_{K}^{2}\left\|\boldsymbol{f}-\Pi_{K}(\boldsymbol{f})\right\|_{0, K}^{2}\right\}^{\frac{1}{2}}\left\|\left(\boldsymbol{u}_{1}-\tilde{\boldsymbol{u}}_{1}, p_{1}-\tilde{p}_{1}\right)\right\|_{h} \\
\leq & \frac{1}{\sqrt{\nu}}\left\{\sum_{K \in \mathcal{T}_{h}} C h_{K}^{4}|\boldsymbol{f}|_{1, K}^{2}\right\}^{\frac{1}{2}}\left\|\left(\boldsymbol{u}_{1}-\tilde{\boldsymbol{u}}_{1}, p_{1}-\tilde{p}_{1}\right)\right\|_{h},
\end{aligned}
$$

and then, dividing by $\left\|\left(\boldsymbol{u}_{1}-\tilde{\boldsymbol{u}}_{1}, p_{1}-\tilde{p}_{1}\right)\right\|_{h}$ we arrive at

$$
\left\|\left(\boldsymbol{u}_{1}-\tilde{\boldsymbol{u}}_{1}, p_{1}-\tilde{p}_{1}\right)\right\|_{h} \leq C \frac{h^{2}}{\sqrt{\nu}}|\boldsymbol{f}|_{1, \Omega},
$$

and both solutions are superclose and the loss of convergence (and consistency) due to the approximation of $\boldsymbol{f}$ by $\Pi_{K}(\boldsymbol{f})$ is of one order smaller than the order of the method.

Now, we test the performance of our methods with the analytical solution

$$
\begin{gathered}
\boldsymbol{u}(x, y)=\left(\begin{array}{c}
-256 x^{2}(x-1)^{2} y(y-1)(2 y-1) \\
256 y^{2}(y-1)^{2} x(x-1)(2 x-1)
\end{array}\right), \\
p(x, y)=150(x-0.5)(y-0.5) .
\end{gathered}
$$

In Figure 1 we depict the convergence history for the method using continuous pressure interpolations and in Figure 2 the convergence history for the method with discontinuous interpolations. We observe that both errors go to zero as predicted by the theory, and the continuous pressure interpolation case achieves even better results. This fact has already been observed in [13] and deserves further investigations.

Finally, in Figure 3 we depict the errors of the method (21) with respect to the parameter $\alpha$. We can observe that, as we said before, the value of $\alpha$ has practically no impact on the 
errors. In fact, when $\alpha$ varies from $10^{-6}$ to 10 we see that the errors vary at most of an order of 10 , and hence our choice of $\alpha=1$ is completely justified.
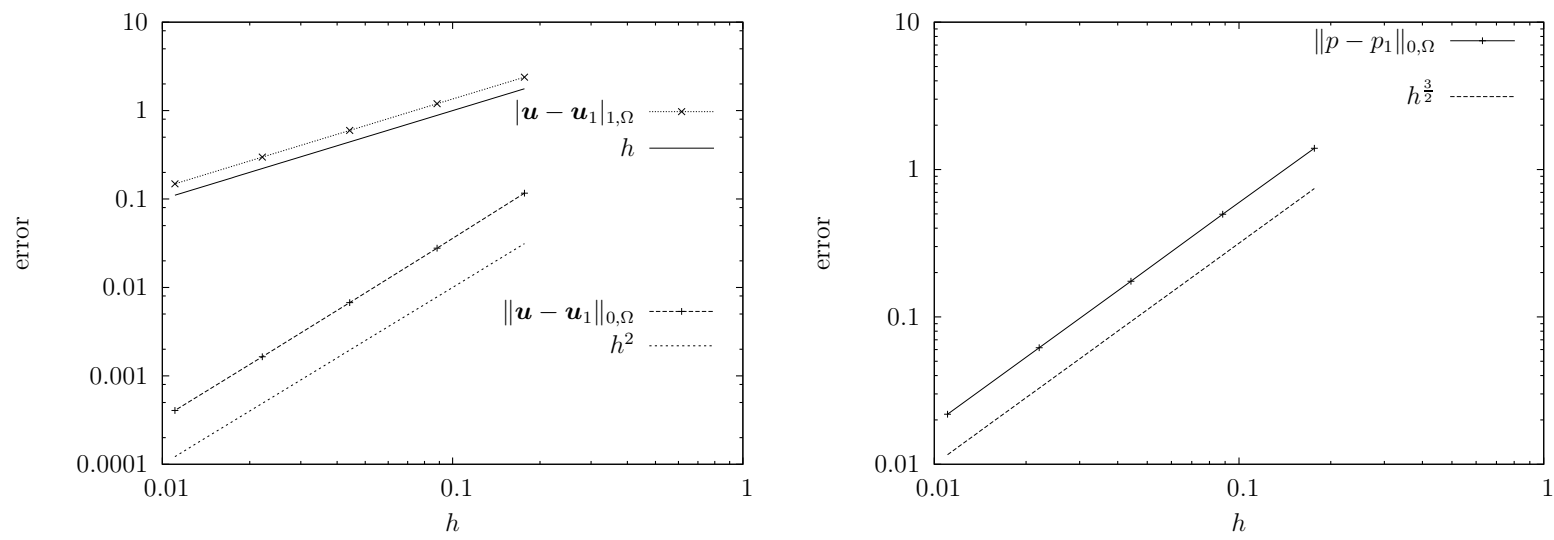

Figure 1. Convergence history of the method (18) (continuous pressures).
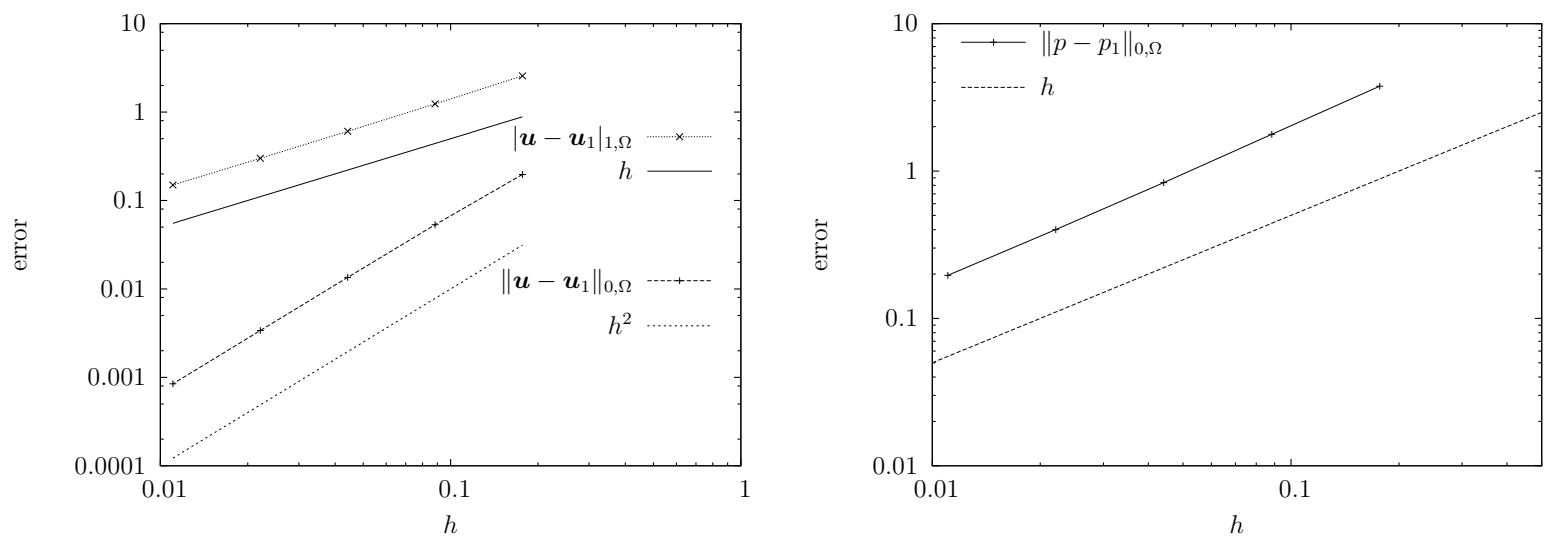

Figure 2. Convergence history of the method (21) (discontinuous pressures). 


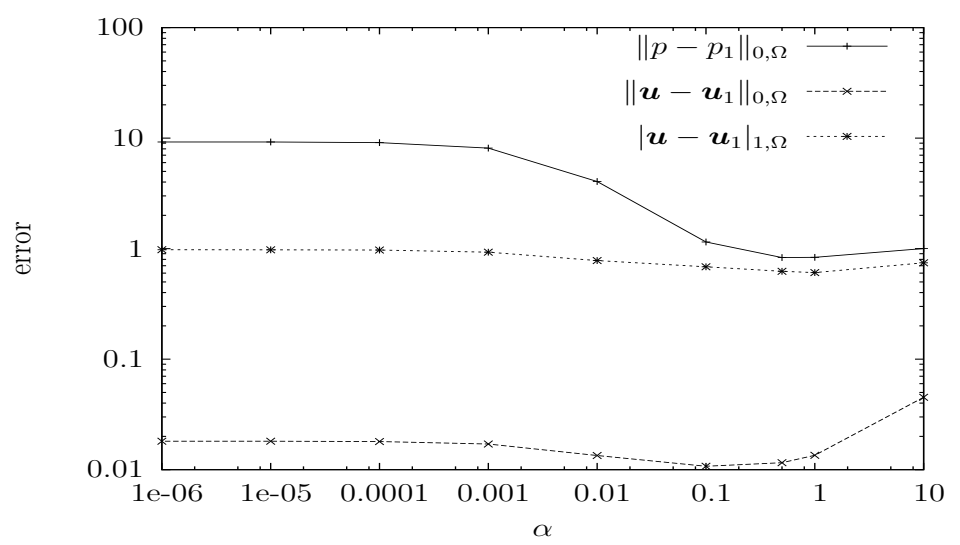

FiguRE 3. Errors of the method (21) with respect to $\alpha$.

\section{Conclusion}

The focus of this work was to establish local projection methods inside an enriching framework relied on residuals. The new way to incorporate them inside a Petrov-Galerkin approach was the key ingredient to achieve stable and consistent new version of LPS (or polynomial projection methods) and still maintain them parameter-free, and without the need of a dual coarser mesh satisfying a macro-element property. We also took advantage of the enhanced space approach to propose a way to recover a locally mass conservative velocity field with and without additional computational cost. Our analysis and numerical validations were limited to piecewise linear continuos and discontinuous interpolation spaces, although the approach is not restricted to them. In the latter case, completely new methods arise for which the denomination of LPS methods is no longer adequate, and for which the Laplacian term no longer vanishes, thus making them strongly consistent. This, as well as extension of the present framework to other problems, such as the Navier-Stokes equation, will be the subject of future research.

\section{ApPEndiX}

Having disregard the term $\sum_{K \in \mathcal{T}_{h}}\left(\boldsymbol{u}_{e}^{D}, \nabla q_{1}\right)_{K}$ in the original method (19) we show here that the procedure does not impact error optimality. This is addressed in the following result:

Theorem 7. Let us suppose that $(\boldsymbol{u}, p)$, solution of $(2)$, belongs to $H^{2}(\Omega)^{2} \times H^{1}(\Omega)$. Furthermore, let $\left(\boldsymbol{u}_{1}, p_{1}\right)$ and $\left(\hat{\boldsymbol{u}}_{1}, \hat{p}_{1}\right)$ be the solutions of (21) and (19), respectively. Then, there 
exists $C, C_{1}>0$, independent of $h$ and $\nu$, such that for $\alpha<C_{1}$ there holds

$$
\left\|\left(\hat{\boldsymbol{u}}_{1}-\boldsymbol{u}_{1}, \hat{p}_{1}-p_{1}\right)\right\|_{h} \leq C h\left(\sqrt{\nu}\|\boldsymbol{u}\|_{2, \Omega}+\frac{1}{\sqrt{\nu}}|p|_{1, \Omega}\right)
$$

Proof. We note the original bilinear form by $\hat{\mathbf{B}}(.$, .), i.e.,

$$
\hat{\mathbf{B}}\left(\left(\boldsymbol{u}_{1}, p_{1}\right),\left(\boldsymbol{v}_{1}, q_{1}\right)\right):=\mathbf{B}\left(\left(\boldsymbol{u}_{1}, p_{1}\right),\left(\boldsymbol{v}_{1}, q_{1}\right)\right)+\sum_{K \in \mathcal{T}_{h}}\left(\boldsymbol{u}_{e}^{D}, \nabla q_{1}\right)_{K}
$$

where $\mathbf{B}(.,$.$) is defined in (22). Stability and consistency for the method (19) must be$ established before heading to prove the error result. For that, we first remark that replacing (66) in (69) and using mesh regularity the following estimate holds for $\boldsymbol{u}_{e}^{D}=\boldsymbol{u}_{e}^{D}\left(\left(\boldsymbol{v}_{1}, q_{1}\right)\right)$ :

$$
\left\|\boldsymbol{u}_{e}^{D}\right\|_{0, K} \leq C h_{K} \sum_{F \subseteq \partial K \cap \Omega} \frac{\alpha h_{F}^{\frac{1}{2}}}{\nu}\left\|\Pi_{F}\left(\llbracket \nu \partial_{\boldsymbol{n}} \boldsymbol{v}_{1}+q_{1} \mathbf{I} \cdot \boldsymbol{n} \rrbracket\right)\right\|_{0, F} \quad \text { for all } K \in \mathcal{T}_{h}
$$

Hence, from the definition of $\hat{\mathbf{B}}(.,$.$) , the Cauchy-Schwarz inequality, (34) and the above$ estimate we get

$$
\begin{aligned}
& \hat{\mathbf{B}}\left(\left(\boldsymbol{v}_{1}, q_{1}\right),\left(\boldsymbol{v}_{1}, q_{1}\right)\right)=\left\|\left(\boldsymbol{v}_{1}, q_{1}\right)\right\|_{h}^{2}+\sum_{K \in \mathcal{T}_{h}}\left(\boldsymbol{u}_{e}^{D}, \nabla q_{1}\right)_{K} \\
& \quad \geq\left\|\left(\boldsymbol{v}_{1}, q_{1}\right)\right\|_{h}^{2}-\sum_{K \in \mathcal{T}_{h}}\left\|\boldsymbol{u}_{e}^{D}\right\|_{0, K}\left\|\nabla q_{1}\right\|_{0, K} \\
& \quad \geq\left\|\left(\boldsymbol{v}_{1}, q_{1}\right)\right\|_{h}^{2}-\sum_{K \in \mathcal{T}_{h}}\left\|\boldsymbol{u}_{e}^{D}\right\|_{0, K} C_{I}^{-1} h_{K}^{-1}\left\|q_{1}-\Pi_{K}\left(q_{1}\right)\right\|_{0, K} \\
& \quad \geq\left\|\left(\boldsymbol{v}_{1}, q_{1}\right)\right\|_{h}^{2}-C \sum_{K \in \mathcal{T}_{h}} \sum_{F \subseteq \partial K \cap \Omega} \frac{\alpha h_{F}^{1 / 2}}{\nu}\left\|\Pi_{F}\left(\llbracket \nu \partial_{\boldsymbol{n}} \boldsymbol{v}_{1}+q_{1} \mathbf{I} \cdot \boldsymbol{n} \rrbracket\right)\right\|_{0, F} C_{I}^{-1}\left\|q_{1}-\Pi_{K}\left(q_{1}\right)\right\|_{0, K} \\
& \quad \geq\left\|\left(\boldsymbol{v}_{1}, q_{1}\right)\right\|_{h}^{2}-\frac{C^{2}}{2 \gamma}\left\|\left(\boldsymbol{v}_{1}, q_{1}\right)\right\|_{h}^{2}-\frac{\alpha \gamma}{2 C_{I}^{2}}\left\|\left(\boldsymbol{v}_{1}, q_{1}\right)\right\|_{h}^{2} \\
& \quad=\left(1-\frac{C^{2}}{2 \gamma}-\frac{\alpha \gamma}{2 C_{I}^{2}}\right)\left\|\left(\boldsymbol{v}_{1}, q_{1}\right)\right\|_{h}^{2},
\end{aligned}
$$

and the coercivity result follows setting $\gamma=C^{2}$ and $\alpha<\left(\frac{C_{I}}{C}\right)^{2}$. In addition, since method (19) is residual-based consistency follows straightforwardly. 
We are ready to prove the error estimate. Using previous results and the estimate for $\boldsymbol{u}_{e}^{D}=\boldsymbol{u}_{e}^{D}\left(\left(\boldsymbol{u}-\boldsymbol{u}_{1}, p-p_{1}\right)\right)$ given in (69) we get

$$
\begin{aligned}
C\left\|\left(\hat{\boldsymbol{u}}_{1}-\boldsymbol{u}_{1}, \hat{p}_{1}-p_{1}\right)\right\|_{h}^{2} & \leq \hat{\mathbf{B}}\left(\left(\hat{\boldsymbol{u}}_{1}-\boldsymbol{u}_{1}, \hat{p}_{1}-p_{1}\right),\left(\hat{\boldsymbol{u}}_{1}-\boldsymbol{u}_{1}, \hat{p}_{1}-p_{1}\right)\right) \\
& =\hat{\mathbf{B}}\left(\left(\boldsymbol{u}-\boldsymbol{u}_{1}, p-p_{1}\right),\left(\hat{\boldsymbol{u}}_{1}-\boldsymbol{u}_{1}, \hat{p}_{1}-p_{1}\right)\right) \\
& =\sum_{K \in \mathcal{T}_{h}}\left(\boldsymbol{u}_{e}^{D}, \nabla\left(\hat{p}_{1}-p_{1}\right)\right)_{K} \\
& \leq \sum_{K \in \mathcal{T}_{h}}\left\|\boldsymbol{u}_{e}^{D}\right\|_{0, K} C_{I}^{-1} h_{K}^{-1}\left\|\hat{p}_{1}-\Pi_{K}\left(\hat{p}_{1}\right)-p_{1}+\Pi_{K}\left(p_{1}\right)\right\|_{0, K} \\
& \leq C h\left(\nu|\boldsymbol{u}|_{2, \Omega}+\frac{1}{\nu}|p|_{1, \Omega}\right)\left\|\left(\hat{\boldsymbol{u}}_{1}-\boldsymbol{u}_{1}, \hat{p}_{1}-p_{1}\right)\right\|_{h},
\end{aligned}
$$

and the result follows by dividing both sides by $\left\|\left(\hat{\boldsymbol{u}}_{1}-\boldsymbol{u}_{1}, \hat{p}_{1}-p_{1}\right)\right\|_{h}$ and reordering constants.

Remark. Convergence rates of order $h$ and $h^{2}$ for the errors $\left\|\hat{p}_{1}-p_{1}\right\|_{0, \Omega}$ and $\left\|\hat{\boldsymbol{u}}_{1}-\boldsymbol{u}_{1}\right\|_{0, \Omega}$ may be accomplish following proof of Theorem 3 and Lemma 4, respectively.

\section{REFERENCES}

[1] R. Araya, G. R. Barrenechea, and F. Valentin, Stabilized finite element methods based on multiscale enrichment for the Stokes problem, SIAM J. Numer. Anal., 44 (2006), pp. 322-348.

$[2]$ - A stabilized finite element method for the Stokes problem including element and edge residuals, IMA Journal of Numerical Analysis, 27 (2007), pp. 172-197.

[3] D. Arnold, F. Brezzi, And M. Fortin, A stable finite element for the Stokes equations, Calcolo, 21 (1984), pp. 337-344.

[4] G. R. Barrenechea, L. P. Franca, and F. Valentin, A Petrov-Galerkin enriched method: a mass conservative finite element method for the Darcy equation, Computer Methods in Applied Mechanics and Engineering, 196 (2007), pp. 2449-2464.

[5] — A symmetric nodal conservative finite element method for the Darcy equation. Preprint 2008-07, Department of Mathematics, University of Strathclyde, 2008.

[6] R. Becker And M. BraAck, A finite element pressure gradient stabilization for the Stokes equations based on local projections, Calcolo, (2001), pp. 173-199.

[7] P. Bochev, C. Dohrmann, and M. Gunzburger, Stabilization of low-order finite elements for the Stokes problem, SIAM J. Numer. Anal., 44 (2006), pp. 82-101.

[8] M. BraACK AND E. Burman, Local projection stabilization for the Oseen problem and its interpretation as Variational Multiscale Method, SIAM J. Numer. Anal., 43 (2006), pp. 2544-2566.

[9] F. Brezzi, L. Franca, T. J. Hughes, and A. Russo, $b=\int g$, Comput. Methods Appl. Mech. Engrg., 145 (1997), pp. 329-339.

[10] F. Brezzi And A. Russo, Choosing bubbles for advection-diffusion problems, Math. Models Methods Appl. Sci., 4 (1994), pp. 571-587. 
[11] E. Burman, Pressure projection stabilizations for Galerkin approximations of Stokes' and Darcy's problem, Numer. Methods Partial Differential Eq., 24 (2008), pp. 127-143.

[12] P. ClÉment, Approximation by finite element functions using local regularization, RAIRO Anal. Numér., (1975), pp. 77-84.

[13] C. Dohrmann And P. Bochev, A stabilized finite element method for the Stokes problem based on polynomial pressure projections, Int. J. Num. Meth. Fluids, 46 (2004), pp. 183-201.

[14] A. Ern and J.-L. Guermond, Theory and Practice of Finite Elements, Springer-Verlag, 2004.

[15] L. P. Franca, A. L. Madureira, and F. Valentin, Towards multiscale functions: enriching finite element spaces with local but not bubble-like functions, Comput. Methods Appl. Mech. Engrg., 194 (2005), pp. 3006-3021.

[16] G. Galdi, An Introduction to the Mathematical Theory of the Navier-Stokes Equations. Vol. I, SpringerVerlag, 1994.

[17] S. Ganesan, G. Matthies, And L. Tobiska, Local projection stabilization of equal order interpolation applied to the Stokes problem, Math. Comp., 77 (2008), pp. 2039-2060.

[18] V. Girault and P. A. Raviart, Finite Element Methods for Navier-Stokes Equations: Theory and Algorithms, vol. 5 of Springer Series in Computational Mathematics, Springer-Verlag, Berlin, New-York, 1986.

[19] T. J. R. Hughes, G. R. Feijoo, L. Mazzei, And J. Quincy, The variational multiscale method - $a$ paradigm for computational mechanics, Computer Methods in Applied Mechanics and Engineering, 166 (1998), pp. 3-24.

[20] L. Payne and H. Weinberger, An optimal Poincaré inequality for convex domains, Arch. Rational Mech. Anal., 5 (1960), pp. 286-292.

[21] R. Pierre, Simple $C^{0}$ approximations for the computation of incompressible flows, Comput. Methods Appl. Mech. Engrg., 68 (1988), pp. 205-227.

[22] H. Roos, M. Stynes, and L. Tobiska, Robust Numerical Methods for Singularly Perturbed Differential Equations, Second Edition, Springer-Verlag, 2008.

Department of Mathematics, University of Strathclyde, 26 Richmond Street, Glasgow G1 1XH, Scotland

E-mail address: grb@maths.strath.ac.uk

Departamento de Matemática Aplicada, laboratório Nacional de ComputaÇão Científica, Av. Getúlio Vargas, 333, 25651-070 Petrópolis - RJ, Brazil

E-mail address: valentin@lncc.br 